\title{
2-15-1994
}

\section{Tidal propagation in strongly convergent channels}

Carl T. Friedrichs

Virginia Institute of Marine Science

David G. Aubrey

Follow this and additional works at: https://scholarworks.wm.edu/vimsarticles

Part of the Oceanography Commons

\section{Recommended Citation}

Friedrichs, Carl T. and Aubrey, David G., Tidal propagation in strongly convergent channels (1994). Journal of Geophysical Research: Oceans, 99(C2), 3321-3336. doi:10.1029/93JC03219

This Article is brought to you for free and open access by the Virginia Institute of Marine Science at W\&M ScholarWorks. It has been accepted for inclusion in VIMS Articles by an authorized administrator of W\&M ScholarWorks. For more information, please contact scholarworks@wm.edu. 


\title{
Tidal propagation in strongly convergent channels
}

\author{
Carl T. Friedrichs ${ }^{1}$ and David G. Aubrey \\ Department of Geology and Geophysics, Woods Hole Oceanographic Institution, \\ Woods Hole, Massachusetts
}

\begin{abstract}
Simple first- and second-order analytic solutions, which diverge markedly from classical views of cooscillating tides, are derived for tidal propagation in strongly convergent channels. Theoretical predictions compare well with observations from typical examples of shallow, "funnel-shaped" tidal estuaries. A scaling of the governing equations appropriate to these channels indicates that at first order, gradients in crosssectional area dominate velocity gradients in the continuity equation and the friction term dominates acceleration in the momentum equation. Finite amplitude effects, velocity gradients due to wave propagation, and local acceleration enter the equations at second order. Applying this scaling, the first-order governing equation becomes a first-order wave equation, which is inconsistent with the presence of a reflected wave. The solution is of constant amplitude and has a phase speed near the frictionless wave speed, like a classical progressive wave, yet velocity leads elevation by $90^{\circ}$, like a classical standing wave. The second-order solution at the dominant frequency is also a unidirectional wave; however, its amplitude is exponentially modulated. If inertia is finite and convergence is strong, amplitude increases along channel, whereas if inertia is weak and convergence is limited, amplitude decays. Compact solutions for second-order tidal harmonics quantify the partially canceling effects of (1) time variations in channel depth, which slow the propagation of low water, and (2) time variations in channel width, which slow the propagation of high water. Finally, it is suggested that phase speed, along-channel amplitude growth, and tidal harmonics in strongly convergent channels are all linked by morphodynamic feedback.
\end{abstract}

\section{Introduction}

In this paper a new asymptotic solution is presented for the barotropic tidal wave in strongly convergent channels. The type of wave described here, which paradoxically exhibits properties of both standing and progressive waves simultaneously, occurs in real tidal estuaries such as the Thames and the Tamar in the United Kingdom and the Delaware in the United States (Figure 1). Like a classical progressive wave, this wave does not appreciably grow or decay along channel, and its phase speed is nearly equal to the frictionless wave speed. Like a classical standing wave, it produces currents which are slack near high and low water. Unlike either wave, however, the dynamic balance which produces this asymptotic solution is strongly frictional. This new solution and its governing equation are markedly different from the classical view of damped tidal cooscillation, yet some of its properties may be confused with classical results. It is useful, therefore, to review briefly the classical approach to tidal propagation in channels.

\footnotetext{
${ }^{1}$ Now at Virginia Institute of Marine Science, School of Marine Science, College of William and Mary, Gloucester Point, Virginia.

Copyright 1994 by the American Geophysical Union.

Paper number 93JC03219.

0148-0227/94/93JC-03219\$05.00
}

\subsection{Classical Tidal Cooscillation}

In a frictionless, prismatic channel of rectangular cross section, the one-dimensional linearized governing equation for elevation $(\zeta)$ reduces to the familiar second-order wave equation [e.g., Ippen, 1966]

$$
\frac{\partial^{2} \zeta}{\partial t^{2}}=c_{0}^{2} \frac{\partial^{2} \zeta}{\partial x^{2}}
$$

where $t$ is time, $x$ is distance, and $c_{0}$ is the frictionless gravity wave speed. With intertidal storage in tidal flats or marsh (Figure 2),

$$
c_{0}=\left(\frac{w}{\bar{b}} g \bar{h}\right)^{1 / 2}=(g \bar{A} / \bar{b})^{1 / 2},
$$

[e.g., Robinson et al., 1983], where $w$ is channel width, $b$ is total estuary width including storage regions, $A$ is channel cross-sectional area, $h=A / w$, and overbars indicate still water values. If the cross section is rectangular, then $w=b$, and (2) reduces to the more familiar relation $c_{0}=(g \bar{h})^{1 / 2}$.

For a sinusoidally forced channel closed at one end, (1) produces a standing wave solution characterized by incident and reflected waves of equal amplitude which individually propagate at $c_{0}$. The incident and reflected waves interact, causing tidal amplitude to vary through nodes and antinodes and producing a relative phase between cross-sectionally averaged velocity $(u)$ and $\zeta$ of $90^{\circ}$. The phase speed, $c$, 


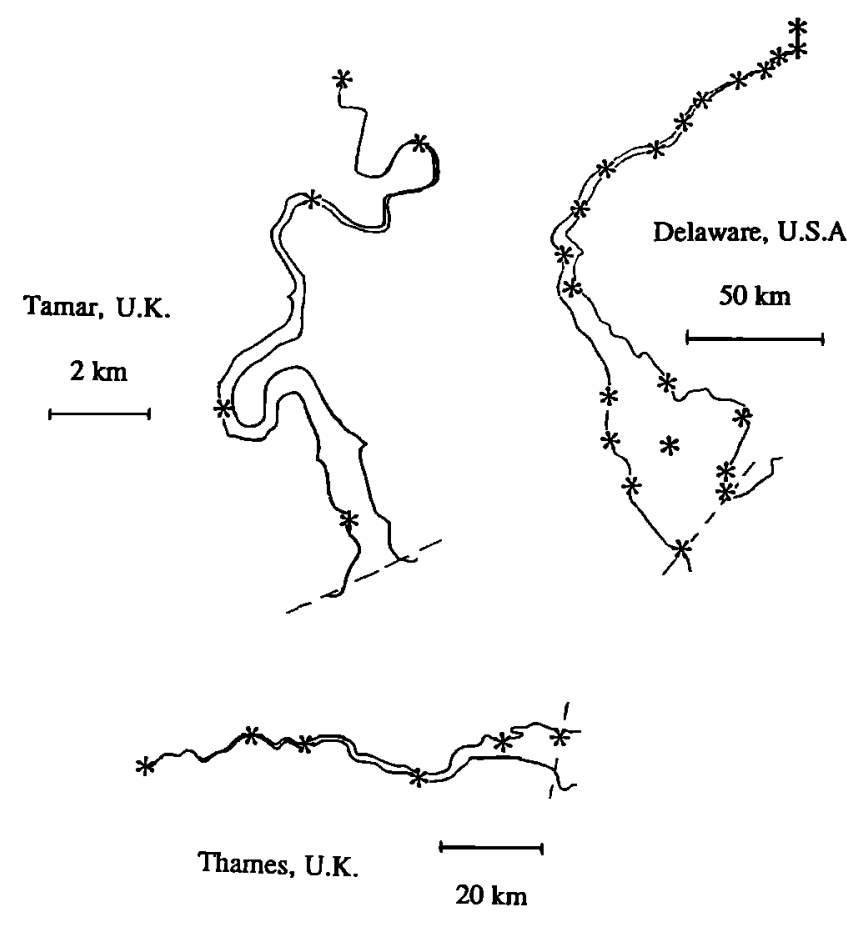

Figure 1. Schematic maps showing locations of tidal elevation stations along the Tamar [George, 1975], Delaware [Parker. 1984], and Thames [Prandle, 1980].

which is due to a superposition of the incident and reflected waves, is infinite. If the channel has a length of exactly onequarter wave, then the incident and reflected waves cancel entirely at the mouth, and resonance occurs within the channel. In a sinusoidally forced channel of infinite length, (1) produces a single constant amplitude progressive wave with $c=c_{0}$, and the relative phase between $\zeta$ and $u$ is $0^{\circ}$.

In his review of tidal dynamics in estuaries, Ippen [1966] provides solutions to (1) for several channel geometries and forcings and also discusses the more "realistic" case of a damped cooscillating tide in a prismatic channel which includes the effects of friction. Inclusion of linear friction transforms (1) into a damped second-order wave equation:

$$
\frac{\partial^{2} \zeta}{\partial t^{2}}+r \frac{\partial \zeta}{\partial t}=c_{0}^{2} \frac{\partial^{2} \zeta}{\partial x^{2}}
$$

where $r$ is a constant friction factor. In a channel closed at one end, the solution to (3) consists of exponentially modified incident and reflected waves which are of equal amplitude at the landward reflection point [e.g., Officer, 1976]. The speeds of the incident and reflected waves are equal and, for weak friction, are only slightly less than $c_{0}$. However, the presence of friction has the effect of weakening resonance and damping the amplitude variation at nodes and antinodes. For an infinite channel, amplitude decays monotonically along channel, and the relative phase of $u$ to $\zeta$ is between $0^{\circ}$ and $45^{\circ}$ depending on the size of $r$. For large $r$, the second term in (3) dominates the first, and (3) ultimately reduces to a time-varying diffusion equation [LeBlond, 1978; Friedrichs and Madsen, 1992].
In strongly convergent channels, the tidal phase speed $(c)$ has been observed to be close to $c_{0}$ [Hunt, 1964; Harleman, 1966]. Thus it is tempting to associate this observation with the dynamics of (1) or (3). However, along strongly convergent tidal channels the relative phase between $\zeta$ and $u$ to has been observed to be nearly constant at $\sim 90^{\circ}$ [ Hunt, 1964; Wright et al., 1973]; i.e., slack currents nearly coincide with high and low water. Equations (1) and (3) can only produce a $\zeta$ - $u$ phase of $90^{\circ}$ throughout a channel if the incident wave is accompanied by a nearly equal amplitude reflected wave. But if that is the case, $c$ (which includes the incident and reflected wave) will be much greater than $c_{0}$. One way to produce realistic first-order solutions for tides in many real tidal channels is to consider both friction and along-channel variation in cross-sectional area.

\subsection{Previous Solutions for Convergent Channels with Friction}

Many authors have derived analytic solutions for convergent channels with friction [Perroud, 1959; Le Floch, 1961; Dronkers, 1964; Hunt, 1964; Prandle and Rahman, 1980; Parker, 1984; Godin, 1988; Jay, 1991]. With two notable exceptions [Hunt, 1964; Jay, 1991], previous studies have stressed similarities between classical damped cooscillation and tidal propagation in weakly convergent channels. In interpreting their solutions as perturbations on classical results, these authors emphasized features such as nodes and antinodes and the importance of incident and reflected waves. With weak convergence, they found propagation of tidal elevation to be qualitatively similar to that described by (3), except that weak convergence tends to counteract the effects of weak friction [Le Floch, 1961; Parker, 1984]. Also, in nonprismatic systems resonant conditions become a function of the shape of the estuary as a whole rather than only a function of length and $c_{0}$ [Prandle and Rahman, 1980].

Hunt [1964] was the first to emphasize the fundamentally different nature of tidal propagation along strongly convergent channels with friction. Hunt solved the linearized one-dimensional equations for exponential convergence with trigonometric functions [c.f., Le Floch, 1961; Parker, 1984; Godin, 1988] and for power-law convergence using Bessel functions [c.f., Perroud, 1959;

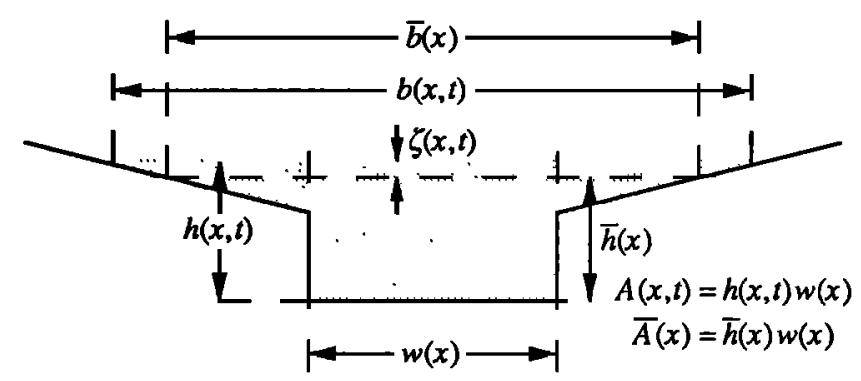

Figure 2. Diagram of an idealized tidal embayment cross section: $b$ is total estuary width (including storage in tidal flats or marsh), $\zeta$ is tidal elevation, $h$ is cross-sectionally averaged channel depth, $w$ is channel width (which is equal to estuary width at low tide), and $A$ is channel cross-sectional area. Overbars indicate time averages. 
Prandle and Rahman, 1980]. Hunt pointed out that unlike solutions for prismatic channels, solutions for strongly convergent channels can produce a progression in tidal phase along the channel while simultaneously maintaining a relative phase of $u$ to $\zeta$ near $90^{\circ}$. He deemphasized the importance of incident and reflected waves by expressing his solutions as single, exponentially modified, forward-propagating waveforms. Finally, Hunt showed his analytic solution to be consistent with observations from the Thames.

Like Hunt [1964], Jay [1991] also emphasized the role of channel convergence in producing a waveform which is fundamentally different from classical damped cooscillation. Jay used a modified Green's Law solution for channels having exponentially varying width and depth, but did not compare analytic results to observations. For strong convergence, Jay also found that a single incident wave in a channel with strongly convergent geometry may mimic a standing wave by having a relative phase of $u$ to $\zeta$ near $90^{\circ}$ without the presence of a reflected wave.

However, neither Hunt [1964] nor Jay [1991] focused on the first-order balances which produce this characteristic behavior. As shown in this paper, doing so allows a simpler first-order solution for tidal propagation which retains and clarifies the most important properties of tides in strongly convergent systems. Jay [1991] specifically examined the asymptotic case of strong convergence with weak friction (which he termed "supercritical convergence"), but his discussion of strong convergence with strong friction was more limited. Also, neither Hunt [1964] nor Jay [1991] recognized the morphological constraint which causes $c$ to be near $c_{0}$ in systems which are both strongly frictional and strongly convergent, namely, that the solution which minimizes along-channel variations in bottom stress also produces $c=c_{0}$.

Part of the difficulty in interpreting results of previous investigators with regard to strongly convergent channels stems from the large number of first-order terms they have all kept in the equations of motion. Previous investigators of convergent systems have assumed (1) that local acceleration contributes to momentum at first order, and (2) that discharge gradients due to velocity variation contribute to continuity at first order. These terms are of secondary importance in the strongly convergent channels that are of interest to this study. Of course, near-resonant, strongly convergent tidal systems do exist where acceleration is more important than friction. Examples include the Gulf of Maine in the United States and the Bristol Channel in the United Kingdom, both of which were examined by Prandle and Rahman [1980]. However, these systems are hundreds of kilometers in length and many tens of meters deep.

In the following section a scaling of the equations of motion is performed which is appropriate to a more common type of strongly convergent tidal channel, namely, those having a mean depth on the order of 10 meters or less. Disadvantageous scalings may have been applied in the past to shallow, strongly convergent channels because of a lack of comparison to observations from real tidal channels during the scaling process. Parker [1984], who applied data from the Delaware Estuary during scaling, did indeed note that friction dominates acceleration in the momentum balance and that discharge gradients due to channel convergence dominate those due to velocity gradients in the mass balance. However, Parker did not take advantage of these relations in his analytic solution.

This new scaling leads to a simpler first-order governing equation which has the form of a first-order wave equation, in contrast to the second-order wave equation which characterizes classical damped cooscillation. The solution includes all the major properties which distinguish tidal waves in shallow, strongly convergent channels, yet is more amenable to conceptual interpretation. Finite amplitude effects, velocity gradients due to wave propagation, and local acceleration (all of which enter the equations at second order) then lead to systematic, interpretable perturbations on the first-order solution.

\section{Scaling of Equations}

The cross-sectionally integrated, one-dimensional equations for a tidal channel with linearly sloping intertidal flats (Figure 2) may be expressed as [Speer and Aubrey, 1985]

$$
\begin{array}{ll}
\text { Continuity } & b \frac{\partial \zeta}{\partial t}=-\frac{\partial}{\partial x}[A u], \\
\text { Momentum } & \frac{\partial u}{\partial t}+u \frac{\partial u}{\partial x}=-g \frac{\partial \zeta}{\partial x}-F,
\end{array}
$$

where $F$ represents bottom friction, and other variables are as defined in section 1 (see the notation list for a summary of all symbols). In addition to the usual assumptions of channelized flow, (4) and (5) assume $u=0$ on the flats [Speer and Aubrey, 1985].

In the following paragraphs, the continuity equation is scaled to determine which terms must be retained at first and second order when examining barotropic tidal propagation in estuaries such as the Thames, Tamar, and Delaware. Results from continuity are then used to scale the momentum equation. For reference, the dimensionless quantities which are assumed to be small in this study are summarized in the appendix.

\subsection{Scaling of Continuity}

For estuaries represented by Figure 2, continuity may be expanded as

$$
\begin{aligned}
& \left(1+\varepsilon_{b} \frac{\zeta}{a}\right) \bar{b} \frac{\partial \zeta}{\partial t}= \\
& -\left(1+\varepsilon_{h} \frac{\zeta}{a}\right)\left\{\frac{\partial \bar{A}}{\partial x}\right\} u-\bar{A} \frac{\partial u}{\partial x}-\mathrm{O}\left(\varepsilon_{h}\right) \bar{A}\left\{\frac{u}{a} \frac{\partial \zeta}{\partial x}+\frac{\partial u}{\partial x}\right\}
\end{aligned}
$$

The small parameters $\varepsilon_{h}$ and $\varepsilon_{b}$ come from finite amplitude and intertidal slope effects and are defined as $\varepsilon_{h}=a / \bar{h}$ and $\varepsilon_{b}$ $=(\bar{b}-w) / \bar{b}$, where $a$ is tidal amplitude, and $w$ is both the width of the channel and the total embayment width at low tide. Overbars indicate time averages such that $h=\bar{h}(1+$ $\left.\varepsilon_{h} \zeta / a\right), b=\bar{b}\left(1+\varepsilon_{b} \zeta / a\right)$, and $A=\bar{A}\left(1+\varepsilon_{h} \zeta / a\right)$. Observed values for $\varepsilon_{h}$ and $\varepsilon_{b}$ are given in Table 1. The first term on the right-hand side of (6) arises from the along-channel 
Table 1. Observed and Computed Tidal and Geometric Properties of Three Tidal Estuaries

\begin{tabular}{|c|c|c|c|}
\hline Parameter & Tamar & Thames & Delaware \\
\hline$L(\mathbf{k m})$ & 21 & 95 & 215 \\
\hline$<a>(m)$ & $2.7 \pm 0.05(a)$ & $2.2 \pm 0.1(b)$ & $0.83 \pm 0.03(c)$ \\
\hline$\langle\bar{h}\rangle(\mathrm{m})$ & $2.9 \pm 0.2(\mathrm{~d})$ & $8.5 \pm 0.7(e, f)$ & $5.8 \pm 0.3(c)$ \\
\hline$E_{h}=\langle a\rangle \mid\langle\bar{h}\rangle$ & $0.94 \pm 0.07$ & $0.26 \pm 0.03$ & $0.143 \pm 0.009$ \\
\hline$\varepsilon_{b}=1-\langle w / \bar{b}\rangle$ & $0.29 \pm 0.09(d)$ & $0.17 \pm 0.02(f)$ & $\sim 0$ \\
\hline$L_{A}(\mathrm{~km})$ & $5.3 \pm 0.2(d)$ & $18.5 \pm 0.8(e, f)$ & $38 \pm 1(c)$ \\
\hline$L_{b}(\mathrm{~km})$ & $4.6 \pm 0.4(d)$ & $25.0 \pm 0.8(\mathrm{e})$ & $40 \pm 1(c)$ \\
\hline$L_{A} / L_{U}$ & $0.033 \pm 0.013(d)$ & $-0.067 \pm 0.073(f, g)$ & $-0.023 \pm 0.025(c, h)$ \\
\hline$L_{\theta}(\mathrm{km})$ & $400 \pm 30(a)$ & $440 \pm 80(b)$ & $365 \pm 7(c)$ \\
\hline$\varepsilon_{A}=L_{A} / L_{\theta}$ & $0.013 \pm 0.001$ & $0.042 \pm 0.007$ & $0.103 \pm 0.004$ \\
\hline$\varepsilon_{A}=k L_{A}=2 \pi L_{A} / L_{\theta}$ & $0.083 \pm 0.007$ & $0.26 \pm 0.05$ & $0.65 \pm 0.02$ \\
\hline$\langle\bar{A} / \bar{b}\rangle(\mathrm{m})$ & $2.0 \pm 0.2(d)$ & $7.0 \pm 0.6(e, f)$ & $5.8 \pm 0.3(c)$ \\
\hline$L_{A} / L_{a}$ & $0.028 \pm 0.002(d, a)$ & $-0.012 \pm 0.032(e, f, b)$ & $0.071 \pm 0.010(c)$ \\
\hline$c / c_{0}=\left(L_{\theta} / T\right)(g \bar{A} / \bar{b})^{-1 / 2}$ & $2.0 \pm 0.2$ & $1.2 \pm 0.2$ & $1.08 \pm 0.04$ \\
\hline$\varepsilon_{\omega}=\left(L_{A} / L_{\theta}\right)\left(c / c_{0}\right)^{2}$ & $0.054 \pm 0.009$ & $0.060 \pm 0.019$ & $0.121 \pm 0.008$ \\
\hline$\varepsilon_{\omega}=k L_{A}\left(c / c_{0}\right)^{2}$ & $0.34 \pm 0.06$ & $0.38 \pm 0.12$ & $0.76 \pm 0.05$ \\
\hline$c_{d}=\frac{3 \pi(\bar{A} / \vec{b})^{2} g}{8 c \varepsilon_{h} L_{A}^{2} \omega}$ & $1.4 \pm 0.2 \times 10^{-3}$ & $4.6 \pm 1.1 \times 10^{-3}$ & $1.7 \pm 0.2 \times 10^{-3}$ \\
\hline$\gamma=\varepsilon_{h}-\varepsilon_{b}$ & $0.65 \pm 0.11$ & $0.090 \pm 0.032$ & $0.143 \pm 0.009$ \\
\hline$\mu=\varepsilon_{\omega}-\varepsilon_{A}=k L_{A}\left(c^{2} / c_{0}^{2}-1\right)$ & $0.26 \pm 0.04$ & $0.11 \pm 0.04$ & $0.11 \pm 0.01$ \\
\hline$\mu=\left(k L_{a}\right)^{-1}$ & $0.34 \pm 0.03$ & $-0.05 \pm 0.12$ & $0.10 \pm 0.02$ \\
\hline
\end{tabular}

Sources are as follows: (a) George [1975]; (b) Prandle [1980]; (c) Parker [1984]; (d) Uncles et al. [1985]; (e) Hunt [1964]; (f) USDMA charts 37145 and 37146; (g) Chantler [1974]; (h) Harleman [1966]. Here \pm indicates standard errors; angle brackets indicate along-channel average. Parameters are further defined in the text and in the notation list.

gradient of cross-sectional area, the second term is due to the along-channel gradient in tidal velocity, and the third term comes from higher-order finite amplitude effects.

In order to scale the terms in (6), $\bar{b}, \bar{A}$, and $u$ are assumed to vary as $e^{-x / L_{b}}, e^{-x / L_{A}}$, and $e^{x / L U}$, where $L_{b}, L_{A}$, and $L_{U}$ are $e$-folding lengths of along-channel variation, and $x=0$ at the forced end of the channel. Figure 3 illustrates the fit of observed $\bar{b}, \bar{A}$ and $U$ to exponential curves (where $U$ is the amplitude of $u$ ), and Table 1 lists observed values for $L_{b}, L_{A}$ and $L_{A} / L_{U}$. Clearly $U$ does not necessarily follow a simple exponential curve over the length of an entire estuary. The main purpose here is to illustrate that $L_{b}$ and $L_{A}$ are of similar magnitude and that both are much less than $L_{U}$ on a system-wide scale (evaluated outside of the immediate vicinity of $x=L$, where a zero tidal flow boundary condition may exist).

For systems described by exponential variation in $\bar{b}, \bar{A}$, and $U,(6)$ can be reexpressed in terms of scales as follows:

$$
\left(1+\varepsilon_{b}\right) \frac{a c \varepsilon_{A}}{(\bar{A} / \bar{b}) U}=\left(1+\varepsilon_{h}\right)+\left(\varepsilon_{U}^{2}+\varepsilon_{A}\right)
$$

where $\varepsilon_{U}{ }^{2}$ and $\varepsilon_{A}$ together indicate the size of $\bar{A} \partial u / \partial x$ relative to $u d \bar{A} / d x$, and the third term on the right-hand side of (6) has been neglected relative to the second. The quantity $\varepsilon_{U}^{2}=$ $L_{A} / L_{U} \mid$ arises from along-channel variation in the amplitude of tidal velocity and is raised to the second power because $L_{A} / L_{U}$ is an order of $\varepsilon$ smaller than $\varepsilon_{h}$ in estuaries of interest to this study (see Table 1). The quantity $\varepsilon_{A}$ comes from along-channel variation in the phase of tidal velocity due to wave propagation.

Depending on the length- and time-scales chosen to parameterize tidal phase, $\varepsilon_{A}$ is defined either as

$$
\varepsilon_{A}=L_{A} / L_{\theta}
$$

or as

$$
\varepsilon_{A}=k L_{A},
$$

where $L_{\theta}$ is the tidal wavelength, and $k=2 \pi / L_{\theta}$ is the corresponding wavenumber. The tidal phase speed in (7) is given by $c=L_{\theta} / T=\omega / k$, where $T$ is the tidal period, and $\omega$ is the tidal radian frequency. The effective tidal wavelength is estimated from observations of along-channel tidal phase (Figure 4); observed values for $L_{\theta}$ and $\varepsilon_{A}$ are presented in Table 1.

In his scaling of continuity for application to the Delaware, Parker [1984] chose to scale $\partial \zeta / \partial t$ and $\partial u / \partial x$ by $a / T$ and $U / L_{\theta}$ which results in the relation for $\varepsilon_{A}$ given by 

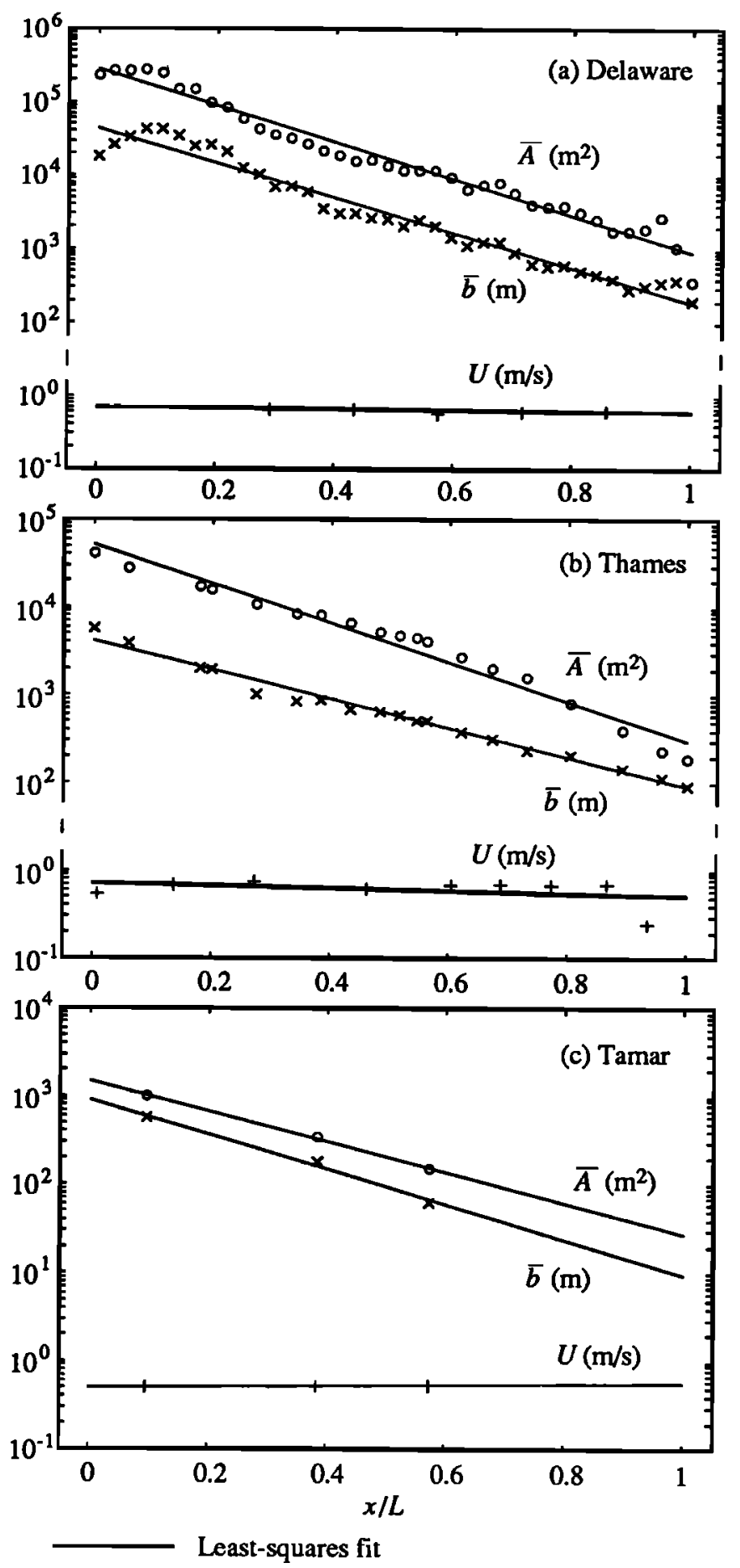

Figure 3. Estimates of channel cross-sectional area at midtide, time-averaged estuary width, and cross-sectionally averaged velocity amplitude as a function of distance along the (a) Delaware $(\vec{A}$ and $\bar{b}$ from Parker [1984], $U$ from Harleman [1966]), (b) Thames ( $\bar{A}$ from Hunt [1964] and USDMA charts 37145 and $37146, \bar{b}$ from Hunt [1964], $U$ from Chantler, [1974]), and (c) Tamar $(\bar{A}, \bar{b}$, and $U$ from Uncles et al. [1985]), along with least squares log-linear regressions.

(8). Equation (8) gives $\varepsilon_{A} \approx 0.10$ for the Delaware (and $\varepsilon_{A}$ $\approx 0.042$ and 0.015 for the Thames and Tamar, respectively), which prompted Parker to conclude that gradients in crosssectional area dominate velocity gradients along the Delaware. However, if one scales $\partial \zeta / \partial t$ and $\partial u / \partial x$ by $\omega a$ and $k U$, then $\varepsilon_{A}$ increases by a factor of $2 \pi$, and $\varepsilon_{A} \approx 0.65$, 0.26 , and 0.091 for the three estuaries. In any case, $u d \bar{A} / d x$ is significantly larger than $\bar{A} \partial u / \partial x$ in (6), even if the latter, more conservative scaling is chosen. Therefore the only term in (6) that can effectively balance $u d \bar{A} / d x$ at first order is $\vec{b} \partial \zeta / \partial t$ (except perhaps for the Delaware if the more conservative scaling is applied). Thus at first order, only these two terms in (6) are retained.

The scaling in this section has shown that in tidal estuaries of interest to this study, along-channel gradients in discharge are dominated by along-channel gradients in cross-sectional area. The next most important contribution to the discharge gradient, at $O\left(\varepsilon_{A}\right)$, is from along-channel variation in the phase of tidal velocity. On a system-wide scale the least important contribution to the discharge gradient, at $\mathrm{O}\left(\varepsilon U^{2}\right)$, is from along-channel gradients in the amplitude of tidal velocity. The above ordering is contrary to classical damped cooscillation in short prismatic channels, which suggests that gradients in the amplitude of velocity should be most important and that gradients in cross-sectional area should be least important.

\subsection{Scaling of Momentum}

For tides and estuaries described in the previous section, momentum may be expressed in terms of scales as

$$
\frac{\varepsilon_{A} C U}{L_{A}}+\frac{U^{2}}{L_{A}}\left(\varepsilon_{U^{2}}+\varepsilon_{A}\right)=\frac{g a}{L_{A}}\left(\varepsilon_{a}^{2}+\varepsilon_{A}\right)+F,
$$

where $\varepsilon_{a}{ }^{2}=L_{A} / L_{a} l$, and $L_{a}$ scales along-channel variations in tidal amplitude in a manner analogous to $L_{U}$ (see Table 1 for observed values). At first order, (7) indicates that

$$
U \approx \frac{a c \varepsilon_{A}}{(\bar{A} / \bar{b})}=\frac{g a c \varepsilon_{A}}{c_{0}^{2}} .
$$

Dropping $\varepsilon_{a}^{2}$ and $\varepsilon_{U}^{2}$ relative to $\varepsilon_{A}$ in (10) and using (11) to eliminate $U$ then yields

$$
\varepsilon_{A} \frac{c^{2}}{c_{0}^{2}}+\frac{\bar{b}}{w} \varepsilon_{h} \varepsilon_{A}{ }^{2} \frac{\mathcal{c}^{2}}{c_{0}^{2}}=1+\frac{F L_{A}}{g a \varepsilon_{A}},
$$

where the terms on the left-hand side of (12) scale local and advective acceleration, respectively, and the terms on the right-hand side of (12) scale pressure gradient and friction.

The observed phase speed, $c$, can be calculated from the known tidal frequency and slope of the observed tidal phase in Figure 4b. Doing so indicates that $c / c_{0}=O(1)$ for the Delaware, Thames, and Tamar (Table 1). Thus $\varepsilon_{\omega}=$ $\varepsilon_{A}\left(c / c_{0}\right)^{2}$, which scales the importance of acceleration relative to the pressure gradient, is less than to much less than one in these three systems. Following the scaling for $\varepsilon_{A}$ suggested by $P$ arker [1984], $\varepsilon_{\omega} \approx 0.12,0.060$, and 0.049 for the Delaware, Thames, and Tamar (Table 1). If one uses the more conservative scaling for $\varepsilon_{A}$ in (9), then $\varepsilon_{\omega} \approx 0.76$, 0.38 , and 0.31 . The only term in (12) that can balance the pressure gradient at lowest order is the friction term (except perhaps for the Delaware if one uses the more conservative scaling for $\varepsilon_{A}$ ).

Thus an important result has been derived: If a tidal channel is strongly convergent (i.e., $\varepsilon_{A} \ll 1$ ) and the observed phase speed is the same order as the frictionless 

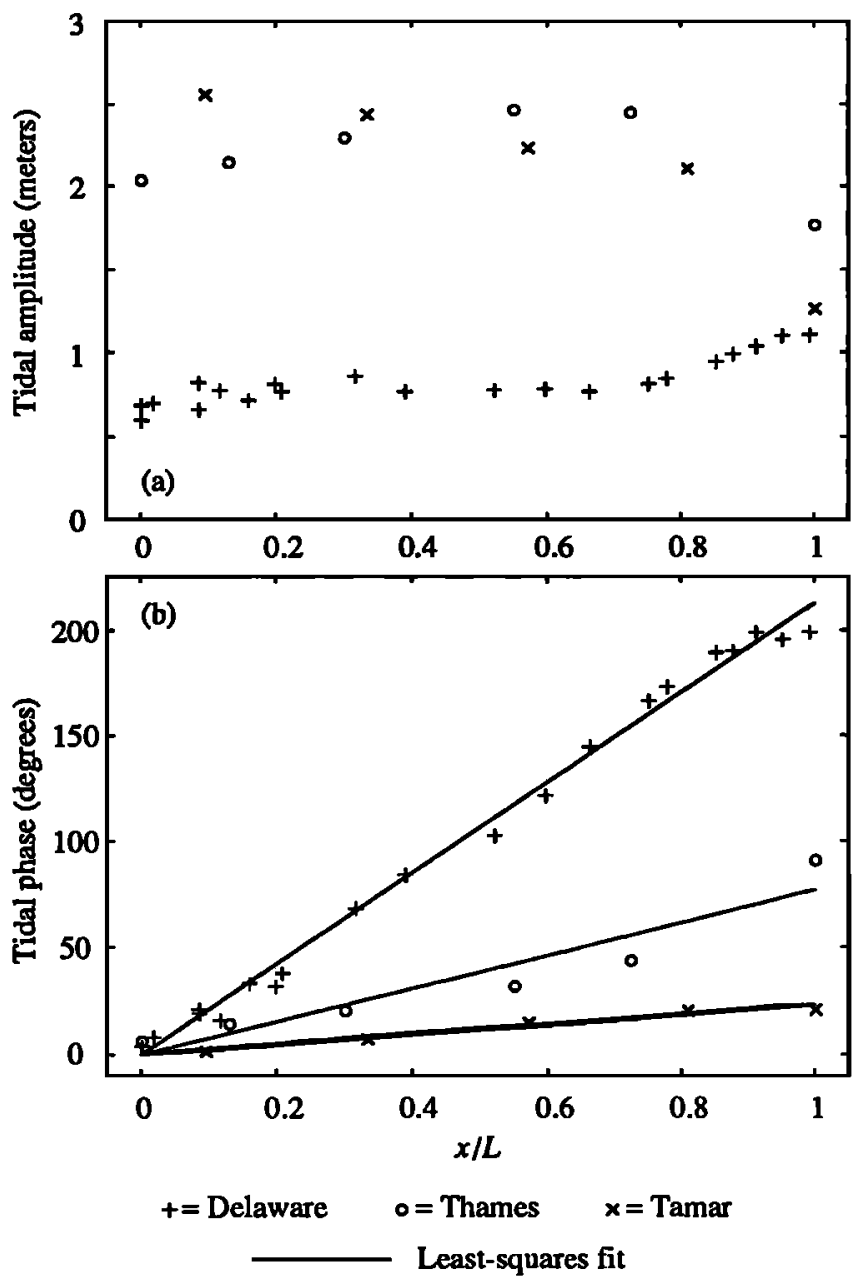

Figure 4. Observed semidiumal surface tide as a function of distance along the Tamar [George, 1975], Thames [Prandle, 1980], and Delaware [Parker, 1984]: (a) amplitude and (b) phase, along with least squares linear regressions. Observations from the Thames and Delaware are of the $\mathbf{M}_{2}$ component. Synoptic observations from the Tamar are limited to a single tidal cycle during spring tide; thus displayed data are derived from harmonic analyses of individual tidal cycles.

wave speed (i.e., $c / c_{0} \approx 1$ ), then the lowest-order momentum balance must be between pressure gradient and friction (i.e., $\left.\varepsilon_{\omega}=\varepsilon_{A}\left(c / c_{0}\right)^{2} \ll 1\right)$. This conclusion has been reached without any a priori knowledge of the depth of the channel, the amplitude of tidal velocity, or the magnitude of the drag coefficient. Furthermore, if $\varepsilon_{A} / \varepsilon_{h} \leq \mathrm{O}(1)$ also holds, then the local acceleration term can be no more important than nonlinearities generated by finite amplitude effects in the continuity equation. Finally, (12) indicates that the advective acceleration term is 3 orders of $\varepsilon$ smaller than friction.

The dominant role of friction suggested above is in contrast with classic solutions for cooscillating tides in prismatic channels, which often neglect friction entirely. Other analytic approximations which consider convergent channel geometry in the presence of friction have always treated friction and local acceleration at the same order. The resulting solutions are often expressed in terms of Bessel equations or repeated variable transformations, which can hamper conceptual interpretation. The scaling presented in this section suggests that by neglecting acceleration at first order, useful insights may be gained toward our understanding of tidal flow in strongly convergent tidal channels. This approach simplifies and clarifies the problem without sacrificing the fundamental physics.

\section{First-Order Solution}

\subsection{Derivation of First-Order Solution}

Retaining only first-order terms, the equations of motion in shallow, strongly convergent tidal channels become

$$
\begin{array}{ll}
\text { Continuity } & \bar{b} \frac{\partial \zeta}{\partial t}=\frac{\bar{A} u}{L_{A}}, \\
\text { Momentum } & 0=-g \frac{\partial \zeta}{\partial x}-F .
\end{array}
$$

In one-dimensional numerical models of channelized tidal flow, the friction term is commonly formulated as [e.g., Speer and Aubrey, 1985]

$$
F=\frac{c_{d}|u| u}{h_{R}} \approx \frac{c_{d}|u| u}{h},
$$

where $c_{d}$ is a time-independent drag coefficient, and the hydraulic radius of the channel, $h_{R}$, is approximately equal to $h$ for channels having $w \gg h$. If velocity is sinusoidal at first order, then (15) can be expanded to second order using Fourier and binomial expansions as follows [e.g., Parker, 1984]:

$$
F=\frac{8}{3 \pi} \frac{c_{d} U}{\bar{h}}\left(1-\varepsilon_{h} \frac{\zeta}{a}\right)\left\{u+\frac{U}{5} \cos \left(3 \omega t-3 \varphi_{u}\right)\right\},
$$

where $U$ and $\varphi_{u}$ are the amplitude and phase angle of $u$. At first order, (16) becomes

$$
F=\frac{8}{3 \pi} \frac{c_{d} U}{\bar{h}} u=r u,
$$

where $r$ is a constant friction factor. The assumption that $r$ is constant in space is only approximately true because of along-channel variations in $c_{d}, U$, and $\bar{h}$.

Combining (13) and (14) then gives

$$
\frac{\partial \zeta}{\partial t}+\frac{c_{0}^{2}}{r L_{A}} \frac{\partial \zeta}{\partial x}=0
$$

which is a first-order wave equation for tidal elevation, markedly different from the second-order wave equation which results from neglecting friction in a prismatic channel. Assuming sinusoidal forcing of amplitude $a$ at $x=0$, the solution to (18) is simply

$$
\zeta=a \cos (\omega t-k x),
$$

and the wave speed is given by

$$
c=\frac{\omega}{k}=\frac{c_{0}^{2}}{r L_{A}} \text {. }
$$


It is interesting to note that the wave number in (19) and (20) is equivalent to the real part of the complex wave number derived by Jay [1991] for his "supercritical convergence" regime.

The solution for velocity is found from (13) to be

$$
u=-U \sin (\omega t-k x)
$$

where $U$ is given by

$$
U=\frac{L_{A} \omega a}{(\bar{A} / \bar{b})} .
$$

Equation (22) is consistent with (11) if $\varepsilon_{A}=k L_{A}$, suggesting (9) is probably the more appropriate scaling for the governing equations.

\subsection{Discussion of First-Order Solution}

The first-order solution for shallow, strongly convergent channels diverges from the conventional view of cooscillating estuary tides. As in a classical standing wave, $u$ and $\zeta$ are out of phase by $90^{\circ}$, yet (19) and (21) individually appear progressive. The first-order solution given by (19) is independent of the length of the tidal estuary, in sharp contrast to the length sensitive quarter-wave resonance of frictionless cooscillation. Furthermore, the solution given by (19) is of constant amplitude, whereas the amplitude of a classical cooscillating tide in a finite channel undulates along channel due to the interaction of incident and reflected waves. The very nature of (18) is inconsistent with a reflected wave because a first-order wave equation allows propagation only in the incident direction.

Equation (20) indicates that as long as $r$ is scaled by the dominant tidal component, the phase speed in shallow, strongly convergent channels is independent of frequency; i.e., the smaller-amplitude wave components are nondispersive at first order. Equations (17), (20), and (22) allow the phase speed to be predicted with the drag coefficient as the only independent parameter:

$$
\frac{\omega}{k}=\frac{3 \pi(\bar{A} / \bar{b})^{2} g}{8 c_{d} \varepsilon_{h} L_{A}^{2} \omega} .
$$

Equation (23) indicates that for the dominant frequency, the wave is dispersive. Thus in channels dominated by diurnal tides, the phase speed will be larger than in an identical channel dominated by semidiumal tides. Equation (23) also allows the observed phase speed to be used to solve for $c_{d}$ directly. The resulting "observed" values for $c_{d}$ are displayed in Table 1 . These $c_{d}$ values compare well with those previously employed in one-dimensional numerical models of strongly convergent tidal estuaries. For example, Uncles and Stephens [1989] used $c_{d}=1.6 \times 10^{-3}$ in modeling the Tamar; Prandle [1974] used $c_{d}$ values between $2.0 \times 10^{-3}$ and $8.7 \times 10^{-3}$ in modeling the Thames; and Parker [1984] used $c_{d}$ values ranging between $2.0 \times 10^{-3}$ and $3.7 \times 10^{-3}$ in modeling the Delaware.

Equation (18) allows only one boundary condition, specified at the seaward end of the channel. Thus (18)-(21) will not be valid in the immediate vicinity of $x=L$ if a zero tidal flow condition exists. However the scaling inherent in
(13) indicates that an upstream no-flow boundary cannot be important to the overall solution. Since the dominant length scale of tidal discharge is $L_{A}$, a no-flow boundary condition at $x=L$ cannot be felt much seaward of $x / L=1-L_{A} / L$, and in tidal estuaries of interest to this study, $L_{A} / L$ is significantly less than 1 (see Table 1). This result has important ramifications concerning the potential effect of tidal barriers. In strongly convergent channels, the installation of a tidal barrier at $x=x_{b}$ should have minimal effect on the tidal signal seaward of $x=x_{b}-L_{A}$. This finding is consistent with Prandle and Rahman [1980], who examined the effect of tidal barriers using Bessel function solutions. Upon introduction of barriers into strongly convergent channels, Prandle and Rahman found the amplitude and phase of elevation and velocity to be altered by only a few percent outside the immediate vicinity of the barrier.

The impact of freshwater discharge on the barotropic tide is also scaled by $L_{A}$. Assuming the freshwater velocity at $x$ $=L$ is less than or equal to $U$, then the ratio of freshwater velocity to total velocity will be negligible for $x / L<\sim 1$ $L_{A} / L$. Wherever possible, observations used in this study are from "low" runoff conditions, further reducing the impact of freshwater discharge on tidal propagation. During high runoff or along channels that are not strongly convergent, river flow will have a more significant effect on tidal propagation throughout the channel [e.g., Godin, 1988; Parker, 1991].

\section{Second-order Solution}

\subsection{Derivation of Second-Order Solution}

At second order (see the appendix), (6) and (7) indicate that the following terms are kept in the continuity equation:

$$
\left(1+\varepsilon_{b} \frac{\zeta}{a}\right) \bar{b} \frac{\partial \zeta}{\partial t}=-\left(1+\varepsilon_{h} \frac{\zeta}{a}\right)\left\{\frac{\mathrm{d} \bar{A}}{\mathrm{~d} x}\right\} u-\bar{A} \frac{\partial u}{\partial x} .
$$

The first-order solution for $u$ may be used in $\bar{A} \partial u / \partial x$ because it is a second-order term. Then to $O(\varepsilon),(24)$ can be reexpressed using binomial expansions as

$$
\left\{1-\left(\varepsilon_{h}-\varepsilon_{b}\right) \frac{\zeta}{a}\right\} \frac{\partial \zeta}{\partial t}=\left(1+i \varepsilon_{A}\right) \frac{c_{0}^{2}}{g L_{A}} u,
$$

where (9) has been used to define $\varepsilon_{A}$. In (25), complex notation has been applied in evaluating $\partial u / \partial x$, and the exponential expressions for $\bar{b}$ and $\bar{A}$ with $L_{A}=L_{b}$ have also been used.

From (10) and (16), the momentum equation at second order is

$$
\begin{aligned}
& \frac{\partial u}{\partial t}=-g \frac{\partial \zeta}{\partial x}- \\
& \frac{8}{3 \pi} \frac{c_{d} U}{\bar{h}}\left(1-\varepsilon_{h} \frac{\zeta}{a}\right)\left\{u+\frac{U}{5} \cos \left(3 \omega t-3 \varphi_{u}\right)\right\} .
\end{aligned}
$$

The cosine term on the right-hand side of (26), which arises from a Fourier expansion of $u l u l$ in (15), is associated with the generation of the third tidal harmonic [e.g., Godin, 1988; Parker, 1991]. Yet one-dimensional numerical solutions for 
tides in shallow channels which employ (15) generally do a poor job of reproducing along-channel variations in $\mathbf{M}_{\mathbf{6}}$ [e.g., Prandle, 1980; Parker, 1984; Friedrichs and Madsen, 1992]. There is little doubt that the friction term is the major source of $\mathbf{M}_{6}$ production within semidiurnal tidal channels. However, (15) and (26) are derived under the assumption that the drag coefficient is time-invariant.

Field observations suggest that in energetic tidal flows, $c_{d}$ can be a complex function of tidal height, tidal velocity, and flow direction. By applying observations to the terms in the one-dimensional momentum equation and solving for the drag coefficient, Lewis and Lewis [1987] and Weisman et al. [1990] found $c_{d}$ to vary by 3-4 times over the tidal cycle, whereas Wallis and Knight [1984] observed an order of magnitude variation in $c_{d}$. Thus the true $\mathrm{M}_{6}$ produced by friction may not closely resemble that predicted by (15) unless $c_{d}$ is more properly represented as time-varying. Because of the limited ability of (15) (with constant $c_{d}$ ) to accurately reproduce $M_{6}$ in shallow, energetic tidal channels, the third harmonic will not be considered further in this study.

Tidal modulation of $c_{d}$ could also affect even harmonics. If modulation of $c_{d}$ were out of phase with modulation of $h_{R}$, net production of even harmonics by the friction term could be reduced. This may be partly why a one-dimensional numerical model of the Thames which used (15) [Prandle, 1980] predicted $\mathrm{M}_{4}$ amplitudes significantly larger than the observed values. Yet in a similar one-dimensional model of the Delaware, Parker [1984] was able to reproduce alongchannel variations in $\mathrm{M}_{4}$ amplitude quite well. When Parker [1984] examined the various sources of $\mathrm{M}_{4}$ in the model, he found that the $\mathrm{M}_{4}$ from nonlinear continuity was 3.7 times larger than the $\mathrm{M}_{4}$ from friction. He stated that the various contributions to $\mathrm{M}_{4}$ had different phases and that the total $\mathrm{M}_{4}$ amplitude was less than the sum of the individual contributions. Thus his model might have reproduced $\mathrm{M}_{4}$ just as well without including tidal modulation of $h$ in the friction term. In this paper we will replace $\varepsilon_{h}$ in (26) with $\delta \varepsilon_{h}$, where $0 \leq \delta \leq 1$, in order to allow leeway in assessing the relative importance of time-varying depth on friction.

After applying the above assumptions, substitution of the first-order solution for tidal velocity into the acceleration term yields

$$
u=-\frac{g}{r}\left(1+i \varepsilon_{\omega}-\delta \varepsilon_{h} \frac{\zeta}{a}\right)^{-1} \frac{\partial \zeta}{\partial x},
$$

where $\varepsilon_{\omega}=\omega / r$ indicates the strength of acceleration relative to friction, and complex notation has been used in evaluating $\partial u / \partial t$. Using (20) and (8), $\varepsilon_{\omega}$ can be reexpressed as $\varepsilon_{\omega}=$ $\left(c / c_{0}\right)^{2} \varepsilon_{A}$, which is the same ratio that scaled the relative importance of acceleration in section 2.2 .

Substituting (27) into (25) to eliminate $u$ then yields a single equation for $\zeta$ at $O(\varepsilon)$ :

$$
\frac{\partial \zeta}{\partial t}+\frac{c}{1+i \mu} \frac{\partial \zeta}{\partial x}=-\frac{c \gamma \zeta}{a} \frac{\partial \zeta}{\partial x},
$$

where the amplitude growth factor is given by

$$
\mu=\varepsilon_{\omega}-\varepsilon_{A}=\varepsilon_{A}\left\{\left(\frac{c}{c_{0}}\right)^{2}-1\right\},
$$

the tidal asymmetry factor is given by

$$
\gamma=(1+\delta) \varepsilon_{h}-\varepsilon_{b},
$$

and $c$ is the first-order phase speed. The left-hand side of (28), like (18), is a first-order wave equation with only one boundary condition at $x=0$. However, there is now a second-order forcing term on the right-hand side. Because the forcing term is second order, it can be evaluated at $O(\varepsilon)$ by substituting in the first-order solution. Application of a trigonometric identity then yields

$$
\frac{\partial \zeta}{\partial t}+\frac{c}{1+i \mu} \frac{\partial \zeta}{\partial x}=-\frac{a c \gamma k}{2} \sin (2 \omega t-2 k x) .
$$

The dominant tidal component is given by the real part of the homogeneous solution to (31):

$$
\zeta_{1}=a \mathrm{e}^{\mu k x} \cos (\omega t-k x) .
$$

The quantity $\zeta_{2}$ is given by the particular solution to (31) plus a second term due to the harmonic present at $x=0(\mu$ may be neglected when evaluating the $O(\varepsilon)$ second harmonic component):

$$
\begin{aligned}
\zeta_{2}= & -\frac{a \gamma}{2} k x \sin (2 \omega t-2 k x) \\
& +a_{2} \cos \left(2 \omega t-2 k x-\theta_{2}\right),
\end{aligned}
$$

where $a_{2}$ and $\theta_{2}$ are the amplitude and phase of $\zeta_{2}$ at $x=0$.

Velocity is found to $O(\varepsilon)$ by substituting $\zeta=\zeta_{1}+\zeta_{2}$ into (25) and again employing trigonometric identities:

$$
\begin{gathered}
u_{1}=-U \mathrm{e}^{\mu k x} \sin \left(\omega t-k x-\varepsilon_{A}\right), \\
u_{2}=\frac{U \gamma}{2}\{\sin (2 \omega t-2 k x)-2 k x \cos (2 \omega t-2 k x)\} \\
-\frac{2 a_{2}}{a} U \sin \left(2 \omega t-2 k x-\theta_{2}\right) .
\end{gathered}
$$

The harmonic components given by (33) and (35) are derived much more easily than previous perturbation expansions for nonlinear tides in shallow tidal channels with friction [e.g., Kreiss, 1957; Shetye and Gouveia, 1992]. The relatively compact form of (33) and (35) stems from the simple dynamics which govern tides in strongly convergent channels.

No set-up of tidal elevation or generation of residual currents occurs at $\mathrm{O}(\varepsilon)$ in strongly convergent channels. As anticipated by Jay [1991], this is because the near $90^{\circ}$ phase difference between elevation and velocity generates relatively little Stokes drift. At spring tide in the Tamar, for example, the cross-sectionally averaged Stokes drift is only about onefifth the amplitude of the cross-sectionally averaged quarterdiurnal velocity component [Uncles et al., 1985; 1986]. Stokes drift occurs when maximum flood and maximum ebb occur at different tidal heights such that maximum velocities are not proportional to maximum transports. In strongly convergent channels, maximum flood and maximum ebb both occur near midtide level. Nonetheless, there is significant set-up of the mean water level in the innermost 
Tamar [George, 1975]. This is largely because low water elevation in the Tamar is kinematically truncated by the elevation of the channel bottom (see discussion in section 4.2).

\subsection{Discussion of Second-Order Solution}

4.2.1. Dominant elevation frequency. Equation (32) describes a unidirectional wave (i.e., with no reflected wave) with the same phase speed as the first-order solution. However, the amplitude is modulated by $e^{\mu k x}$, where the amplitude growth factor, $\mu$, is given by (29). This result is consistent with the observations in Figure 4 (especially for the Delaware) which indicate along-channel phase variation is more strongly linear than along-channel amplitude variation.

The exponential modulation of tidal amplitude at second order is due to the combined, partially canceling effects of (1) inertia relative to friction, which is represented by $\varepsilon_{\omega}$, and (2) limited convergence, which is represented by $\varepsilon_{A}$. Conceptually, nonzero $\varepsilon_{\omega}$ indicates inertia has the potential to overcome frictional damping and increase tidal amplitude by causing an along-channel convergence of energy. This phenomenon is analogous to Green's law in the frictionless, weak convergence limit and has previously been termed "topographic funneling" [Jay, 1991]. Nonzero $\varepsilon_{A}$ or "limited convergence" (i.e., a tendency toward a prismatic channel) counteracts topographic funneling because energy is concentrated less effectively if convergence is weak.

If the amplitude growth factor is positive $(\mu>0)$, then inertia overcomes damping due to friction and limited convergence, and tidal amplitude grows along channel. If the amplitude growth factor is negative $(\mu<0)$, damping due to limited convergence and friction overshadows inertia, and amplitude decays. Because $\mu$ is smaller than either $\varepsilon_{\omega}$ or $\varepsilon_{A}$, the second-order solution for elevation is more like the firstorder solution than might be predicted from the size of $\varepsilon_{\omega}$ or $\varepsilon_{A}$ alone. Thus the applicability of the first-order scaling, if based on the size of $\mu$, is extended. This is why the firstorder solution represents the tide in the Delaware reasonably well, even though $\varepsilon_{A}$ and $\varepsilon_{\omega}$ are relatively large. If $\mu=0$, these two second-order effects cancel entirely, and the solution at the dominant frequency is identical to the firstorder case. In strongly convergent channels, the observed exponential variation in the amplitude of tidal elevation, described by the e-folding length $L_{a}$, should be related to the amplitude growth factor by $\mu=\left(k L_{a}\right)^{-1}$. Table 1 lists $\left(k L_{a}\right)^{-1}$ for the Tamar, Thames, and Delaware, and the correspondence to (29) for the Delaware and Thames is reasonably consistent within error bars.

Along the Tamar, $\mu=\varepsilon_{\omega}-\varepsilon_{A}=0.22 \pm 0.05$ suggests that amplitude should increase with distance along channel, yet Figure 4a indicates that amplitude decreases. However, the observed spring tidal amplitude along the Tamar is not entirely dynamic. Because the amplitude to depth ratio in the upper reaches of the Tamar is near unity, low-water elevation in the upper Tamar is kinematically constrained by the elevation of the channel bottom, and the tidal curve is truncated around low water [George, 1975]. If the dynamic amplitude along the Tamar is redefined as local high-water elevation minus midtide elevation at the seaward gauge (both of which were measured relative to a common datum by George), amplitude is then observed to increase along channel. Figure 5 displays (32) superimposed on observations of high-water propagation along the Tamar. The only parameter adjusted in the analytic solution is the friction factor, $r$, which determines $k$ and $\mu$ via (20) and (29).

The prediction of second-order amplitude and phase variation along the Thames and Delaware can be improved by dividing each observed channel into several individual, exponentially varying segments. Because (32) describes a unidirectional wave, the change in amplitude and phase along segment $j$ is given directly by

$$
\begin{aligned}
\left|\eta_{1, j}\right| & =\left|\eta_{1, j-1}\left(L_{j-1}\right)\right| \mathrm{e}^{\mu j k_{j} x_{j}}, \\
\varphi_{\eta 1, j} & =k_{j} x_{j}+\varphi_{\eta 1, j-1}\left(L_{j-1}\right),
\end{aligned}
$$

where $\mu_{j}$ and $k_{j}$ are calculated from the along-channel geometry of each segment, and $x_{j}=0$ at the beginning of
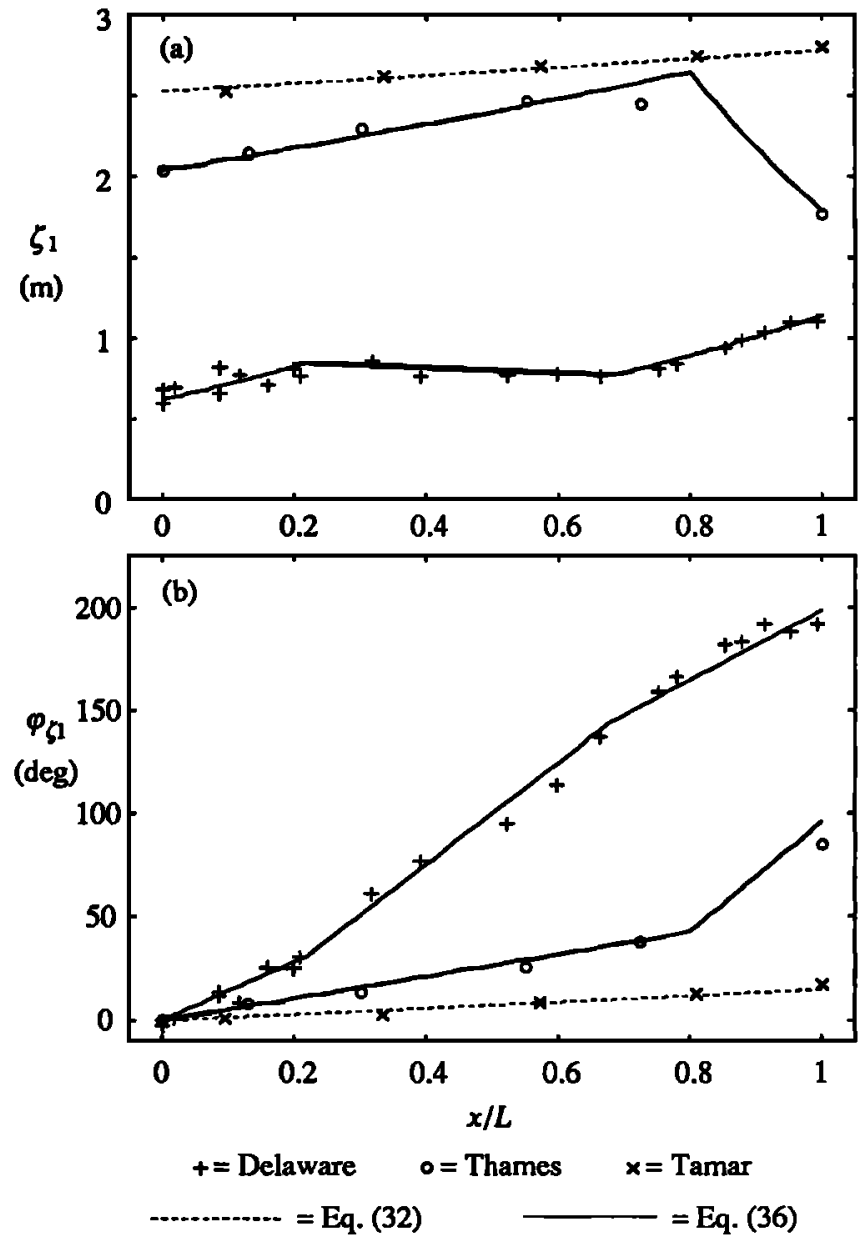

Figure 5. Observed semidiumal surface tide as a function of distance along the Tamar [George, 1975], Thames [Prandle, 1980], and Delaware [Parker, 1984], along with predictions given by the second-order solutions: (a) amplitude, (b) phase. Because low-water elevation in the upper Tamar is kinematically truncated around low water, observations for the Tamar are based on the elevation and phase of high water. In calculating $r$ for the second-order solutions, $c_{d} U=1.1 \times 10^{-3} \mathrm{~m} / \mathrm{s}$ for both the Tamar and the Delaware and $c_{d} U=2.8 \times 10^{-3} \mathrm{~m} / \mathrm{s}$ for the Thames. 
each segment. The only freely determined parameter is $r$, which is used to determine $k_{j}$ and $\mu_{j}$ via (20) and (29). The boundary condition for each segment is simply the amplitude and phase at the end of the previous segment. Unlike segmented solutions to wave equations containing second derivatives [e.g., Dronkers, 1964; Jay, 1991], there is no need to match a reflected wave at the boundary.

Along-channel variations in observed $\left|\eta_{1}\right|$ and $\varphi_{\eta 1}$ (see Figure 4) suggest that an improved representation of the Thames requires at least two exponentially fit segments, whereas the Delaware requires at least three. Table 2 displays values of $L_{A j}, \varepsilon_{A j}$, and $\mu_{j}$ calculated for each segment. Where $\mu_{j}>0$, tidal amplitude locally increases with distance along channel, and where $\mu_{j}<0$, tidal amplitude locally decreases (Figure 5). Successful reproduction of second-order perturbations in along-channel tidal amplitude and phase suggests that these observed undulations are largely the result of deviations from a single exponentially convergent geometry. They are not entirely due to interactions between incident and reflected waves, as commonly presumed through application of classical damped cooscillation. Equation (36) reproduces these undulations yet includes no reflected wave.

Other authors have discussed the control of tidal amplitude by channel convergence and friction in a more qualitative fashion [Allen et al., 1980; Salomon and Allen, 1983; Nichols and Biggs, 1985]. These authors define an estuary to be (1) "hypersynchronous" if strong convergence dominates friction and amplitude grows along channel, (2) "hyposynchronous" if friction dominates convergence and amplitude decays, or (3) "synchronous" if convergence and friction balance and amplitude is constant. For strongly convergent estuaries of interest to this study, a hypersynchronous estuary corresponds to $\mu>0$, a hyposynchronous estuary corresponds to $\mu<0$, and a synchronous estuary corresponds to $\mu=0$. Allen et al. [1980], Salomon and Allen [1983], and Nichols and Biggs [1985] all cite the work of Le Floch [1961], who examined tidal propagation with friction in an infinite tidal channel of constant depth, rectangular cross-section, and width decreasing like $e^{-x / L b}$. For such a channel, Le Floch found that tidal amplitude remains constant with distance (i.e., the channel is "synchronous") if $L_{b}=c_{0} / r$.

Equation (29) indicates that in a synchronous channel, $\varepsilon_{\omega}$ $=\varepsilon_{A}$ and $c=c_{0}$. Using the definitions $\varepsilon_{\omega}=\omega / r, \varepsilon_{A}=k L_{A}$, and $c=\omega / k$, it is easy to show that for a synchronous tidal estuary, $L_{A}=c_{0} / r$. This result is consistent with Le Floch [1961], since in systems of interest to this study, $L_{A} \approx L_{b}$.
Nonetheless, Le Floch emphasized similarities between classical cooscillation and tidal propagation in convergent channels. He stressed that his synchronous solution only applied to idealized infinite channels, and that [Le Floch, 1961, p.363] "In a real estuary it is always necessary to calculate the reflected wave." As shown in this study, however, strongly convergent channels represent an asymptote where it is not necessary to calculate the reflected wave, even in a channel of finite length. Outside of the immediate vicinity of $x=L$ (where boundary conditions on $U$ may invalidate the required scaling), tidal propagation in strongly convergent channels is inherently inconsistent with the presence of a reflected wave. Equation (31), which is a first-order wave equation, allows propagation only in the incident direction.

4.2.2. Dominant velocity frequency. Like the second-order solution for $\zeta_{1},(34)$ also describes a purely unidirectional wave, with the same phase speed as the firstorder wave and with amplitude exponentially modulated by the amplitude growth factor. On a system-wide scale, the value of $\mu$ appropriate to the Tamar, Thames, and Delaware is only slightly greater than zero, thus $\left|u_{1}\right|$ should vary only weakly along the length of these systems. This prediction is consistent with observations of cross-sectionally averaged velocity presented in Figure 3. Chantler [1974], who examined velocity amplitude along six tidal channels, and Friedrichs [1993], who examined velocities in 18 tidal systems, found that stable tidal channels are characterized by velocity amplitudes which are nearly uniform in space. Thus the observed tendency for real channels to have $\mu \approx 0$ may be closely linked to natural patterns of morphologic evolution.

If the amplitude growth factor is significantly different from zero, system-wide gradients in the magnitude of velocity and of bottom stress will exist, and the large-scale channel form may not be stable. Because the bottom stress associated with resuspension is typically higher than that associated with deposition (due to "scour" and "settling" lags, Postma [1967]), system-wide gradients in bottom stress will favor net sedimentation in areas of low stress or net erosion in areas of high stress, and sedimentation or erosion will favor adjustment of $\mu$ toward zero. Equation (29) indicates that as $\mu \rightarrow 0, c \rightarrow c_{0}$. Thus as morphologic adjustment causes tidal velocity to become uniform along the length of the channel, the tidal phase speed will be constrained to be close to the frictionless wave speed. This explains why $c \approx c_{0}$ in strongly convergent tidal channels, even though the dynamics in these systems are strongly frictional. The above argument is admittedly an

Table 2. Properties of Exponentially Convergent Segments of Tidal Estuaries

\begin{tabular}{lcccccc}
\hline Estuary & Segment & $x / L$ & $L_{A}, \mathrm{~km}$ & $\langle\bar{h}>, \mathrm{m}$ & $\varepsilon_{A}=k L_{A}$ & $\mu$ \\
\hline Thames & 1 & $0-0.8$ & 22 & 9.5 & 0.21 & 0.35 \\
& 2 & $0.8-1$ & 12 & 3.2 & 0.61 & -0.42 \\
Delaware & 1 & $0-0.22$ & 40 & 6.8 & 0.46 & 0.58 \\
& 2 & $0.22-0.68$ & 44 & 5.3 & 0.87 & -0.048 \\
& 3 & $0.68-1$ & 33 & 5.6 & 0.45 & 0.41 \\
\hline
\end{tabular}


oversimplification in that it does not consider the role of tidal asymmetries. Morphodynamic feedback between tidal amplitude growth and tidal harmonics is discussed further in section 4.2.4.

Another important feature of (34) is the role played by $\varepsilon_{A}$ in determining the phase by which $u_{1}$ leads $\zeta_{1}$ :

$$
\varphi_{\zeta 1}-\varphi_{u 1}=\frac{\pi}{2}-\varepsilon_{A} .
$$

where the phase of $u_{1}$ is found by reexpressing $u_{1}$ as $\left|u_{1}\right| \cos$ $\left(\omega t-\varphi_{u 1}\right)$. If $\varepsilon_{A}$ is vanishingly small, i.e., if along-channel convergence is infinitely strong, then $u_{1}$ leads $\zeta_{1}$ by $90^{\circ}$, which is identical to the first-order case. For larger $\varepsilon_{A}, u_{1}$ leads $\zeta_{1}$ by a smaller amount. Using $\varepsilon_{A}=k L_{A}$ and (23), (37) may be reexpressed as

$$
\varphi_{\zeta 1}-\varphi_{u 1}=\frac{\pi}{2}-\frac{8}{3 \pi} \frac{c_{d} \varepsilon_{h} \omega^{2} L_{A}{ }^{3}}{g(\bar{A} / \bar{b})^{2}}
$$

Equation (38) indicates that in a strongly convergent tidal channel, $\varphi_{\zeta 1}-\varphi_{u 1}$ responds primarily to local geometric conditions, in sharp contrast to the classical view of frictionally damped cooscillating tides in finite channels. Classical theory indicates that in a channel of finite length, the relative phase of velocity should be a strong function of $x / L$. For a frictionally damped cooscillating tide in a prismatic channel, $\varphi_{\zeta 1}-\varphi_{u 1} \rightarrow 90^{\circ}$ as $x \rightarrow L$ because of complete reflection at the head, and $\varphi_{\zeta 1}-\varphi_{u 1}$ decreases as $x$ $\rightarrow 0$ as the reflected wave becomes more damped with respect to the incident wave.

Figure 6 displays observations of $\varphi_{\zeta 1}-\varphi_{u 1}$ (based on point measurements of $u_{1}$ ) as a function of $\varepsilon_{A}$ superimposed on (37). Complete tidal cycles of simultaneous velocity and elevation are available for the Tamar at $x / L=0.33$ and 0.57 [George, 1975]. The relative phase of velocity along the Delaware is available at $x / L=0$ (at the center of the bay mouth) [Münchow et al., 1992] and $x / L=0.39$ [Parker, 1984], which are in the first and second "segments" of the estuary, respectively (see Table 2 ). The three velocity records for the Thames are from $x / L=0,0.38$ and 0.73 [Hunt, 1964], all of which are in the first segment. The agreement between observations and (37) is quite good for the Delaware, the Tamar, and for two of the three observations from the Thames. The poorly matched point is for observations at $x / L=0.73$ in the Thames, which is relatively near the transition to segment 2 . The data point in parentheses is $\varphi_{\zeta 1}-\varphi_{u 1}$ at $x / L=0.73$ plotted versus the $\varepsilon_{A}$ value appropriate to segment 2 .

4.2.3. Elevation harmonics and tidal asymmetry. The mechanism which produces tidal asymmetry in strongly convergent channels may be understood conceptually if (20) is allowed to vary with tidal height as a function of overall channel depth and estuary width:

$$
\begin{aligned}
& c(t) \approx \frac{c_{0}^{2}(t)}{r(t) L_{A}} \approx \\
& \quad \frac{g w \bar{h}\left(1+\varepsilon_{h} \zeta / a\right)}{\bar{r}\left(1-\delta \varepsilon_{h} \zeta / a\right) L_{A} \bar{b}\left(1+\varepsilon_{b} \zeta / a\right)} \approx \bar{c}(1+\gamma \zeta / a) .
\end{aligned}
$$

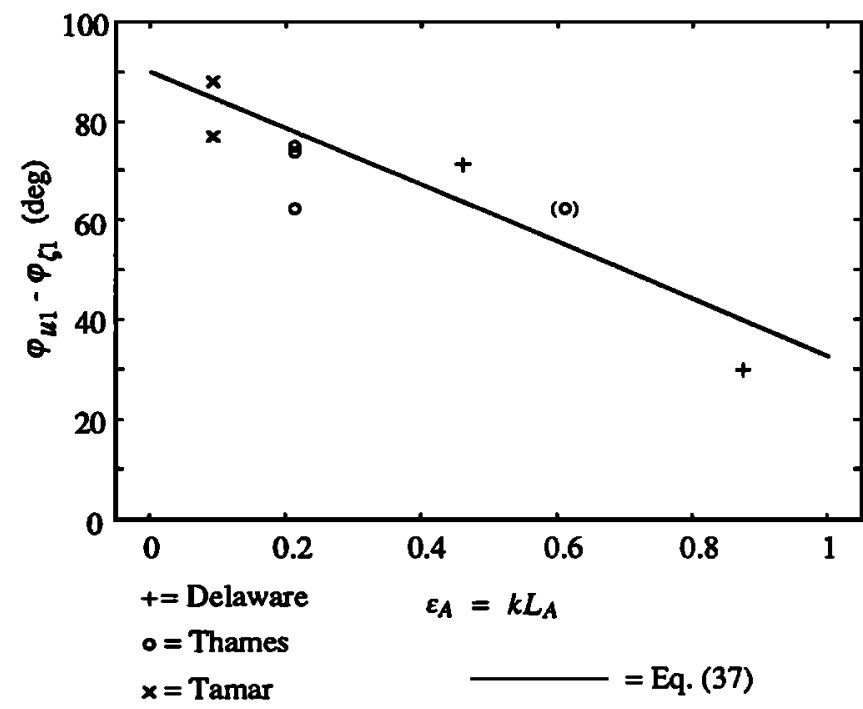

Figure 6. Observed phase of velocity relative to elevation at the dominant tidal frequency for the Tamar [George, 1975], Thames [Hunt, 1963], and Delaware [Parker, 1984; Münchow et al., 1992], along with predictions given by the second-order "segmented" solution as a function of $\varepsilon_{A}=k L_{A}$. The poorly matched point for the Thames is in segment 1 , but relatively near the transition to segment 2 . Parentheses indicate the same point plotted using the geometry of segment 2 .

If $\gamma>0, c(t)$ is greater around high water than it is around low water; high water "catches up" with low water, and the rising tide is of shorter duration. If $\gamma<0, c(t)$ is greater around low water, and the result is a shorter-falling tide. The asymmetry factor, $\gamma=(1+\delta) \varepsilon_{h}-\varepsilon_{b}$, synthesizes the competing effects of time variations in channel depth and time variations in total estuary width. If $\varepsilon_{h} \approx O(1) \gg \varepsilon_{b}$, a much smaller channel cross-sectional area is available around low water to pass a given volume of water (due to nonlinear continuity effects), and propagation of low water is slower. In addition, the depth dependence of $r$ causes the friction term to be stronger around low water, further slowing its propagation. If $\varepsilon_{b} \approx O(1) \gg \varepsilon_{h}$, a much larger volume of water around high water must pass though a given channel cross section, and propagation of high water is slower.

The Tamar, Thames, and Delaware all have positive asymmetry factors and rising tides of shorter duration. Estuaries with $\gamma<0$ are also common, although they tend not to be strongly convergent [Friedrichs and Madsen, 1992]. Analogous results based on numerical modeling of shallow prismatic channels are provided by Speer and Aubrey [1985] and Friedrichs and Aubrey [1988]. Other authors have attributed observations of shorter-rising tides in shallow convergent channels directly to time variation of $c_{0}$, as would be the case in an infinite, prismatic, frictionless channel [McDowell and O'Connor, 1977; Salomon and Allen, 1983]. Since the observed phase speed in real tidal channels is often near $c_{0}$, it is not surprising that previous authors have looked to the frictionless wave speed for an explanation. From (39), however, it is clear that perturbations around $c$ due to time-varying depth and width in shallow, strongly convergent channels are proportional to $c_{0}^{2}$, not $c_{0}$. Furthermore, the dynamics involved are related 
to strong convergence and strong friction and are distinctly different from propagation in prismatic frictionless channels.

The net effect of different propagation speeds around high and low water is represented in the second-order solution by the superposition of $\zeta_{2}$ and $\zeta_{1}$. The amplitude ratio $\left|\zeta_{2} / \zeta_{1}\right|$ indicates the absolute distortion of the tidal curve and, for a given relative phase, increases directly with the degree of asymmetry [Friedrichs and Aubrey, 1988]. Neglecting $\mu$, the ratio of $\zeta_{2}$ to $\zeta_{1}$ is found from (32) and (33) to be

$$
\left.\left|\frac{\zeta_{2}}{\zeta_{1}}\right|=\mid \frac{\gamma^{2}}{4} k^{2} x^{2}-\frac{a_{2}}{a} \gamma k x \sin \theta_{2}+\frac{a_{2}^{2}}{a^{2}}\right\}^{1 / 2} .
$$

For $a_{2}=0,(40)$ reduces simply to

$$
\left|\frac{\zeta_{2}}{\zeta_{1}}\right|=\frac{|\gamma|}{2} k x \text {. }
$$

Equations (40) and (41) predict that asymmetry will increase linearly along channel (assuming $a_{2}$ is small), which is qualitatively consistent with the effect of different phase speeds around high and low water in (39).

The phase of $\zeta_{2}$ relative to $\zeta_{1}$, defined by $2 \phi_{\zeta_{1}}-\varphi_{\zeta 2}$, indicates whether an asymmetric tidal cycle has a rising tide of shorter duration or a falling tide of shorter duration [e.g., Friedrichs and Aubrey, 1988]. Shorter-rising tides have $2 \varphi_{\zeta_{1}}-\varphi_{\zeta_{2}}$ between 0 and $\pi$ (or $0^{\circ}$ and $180^{\circ}$ ), whereas shorter-falling tides have $2 \varphi_{\zeta 1}-\varphi_{\zeta 2}$ between $-\pi$ and 0 (or $-180^{\circ}$ and $0^{\circ}$ ). Neglecting $\mu, 2 \varphi_{\zeta 1}-\varphi_{\zeta 2}$ is found from (32) and (33) to be

$$
\begin{aligned}
& 2 \varphi_{\zeta 1}-\varphi_{\zeta 2}=2 k x+ \\
& \arctan \left\{\frac{\frac{\gamma}{2} k x \sin \left(\frac{\pi}{2}-2 k x\right)-\frac{a_{2}}{a} \sin \left(\theta_{2}+2 k x\right)}{\frac{\gamma}{2} k x \cos \left(\frac{\pi}{2}-2 k x\right)+\frac{a_{2}}{a} \cos \left(\theta_{2}+2 k x\right)}\right\} .
\end{aligned}
$$

where the four quadrant arctangent is evaluated. For $a_{2}=0$, (40) reduces to

$$
2 \varphi_{\zeta 1}-\Phi_{\zeta_{2}}=\frac{\pi}{2} \frac{\gamma}{|\gamma|}
$$

For relatively small $a_{2},(42)$ and (43) predict the tide will be shorter rising if $\gamma$ is positive and shorter falling if $\gamma$ is negative, which is the same pattern predicted by (39). As internally generated $\zeta_{2}$ becomes more important, $2 \varphi_{\zeta 1}-\varphi_{\zeta 2}$ asymptotically approaches $\pm 90^{\circ}$.

Figure 7 compares (40) and (42) to observations of $1 \zeta_{2} / \zeta_{1} \mid$ and $2 \varphi_{\zeta 1}-\varphi_{\zeta 2}$ using first-order values for $k$ derived from Figure $4 \mathrm{~b}$ (see Table 1). Observations of $\left|\zeta_{2} / \zeta_{1}\right|$ and $2 \varphi \zeta_{1}$ $\varphi_{\zeta 2}$ for the Tamar and Delaware are from data of George [1975] and Parker [1984]. Observations of $M_{2}$ and $M_{4}$ phases for the Thames presented by Prandle [1980], however, are relative to separate constants and thus are in a form inconsistent with the application of (40), (42) and (43). Less extensive observations, derived from harmonic analyses of individual tidal cycles in Hunt [1964], are displayed instead. Since Hunt's observations are for spring tides, $\varepsilon_{h}$ and $\varepsilon_{b}$ are each slightly higher (at 0.30 and 0.20 ) than the mean values appropriate to Prandle's observations.
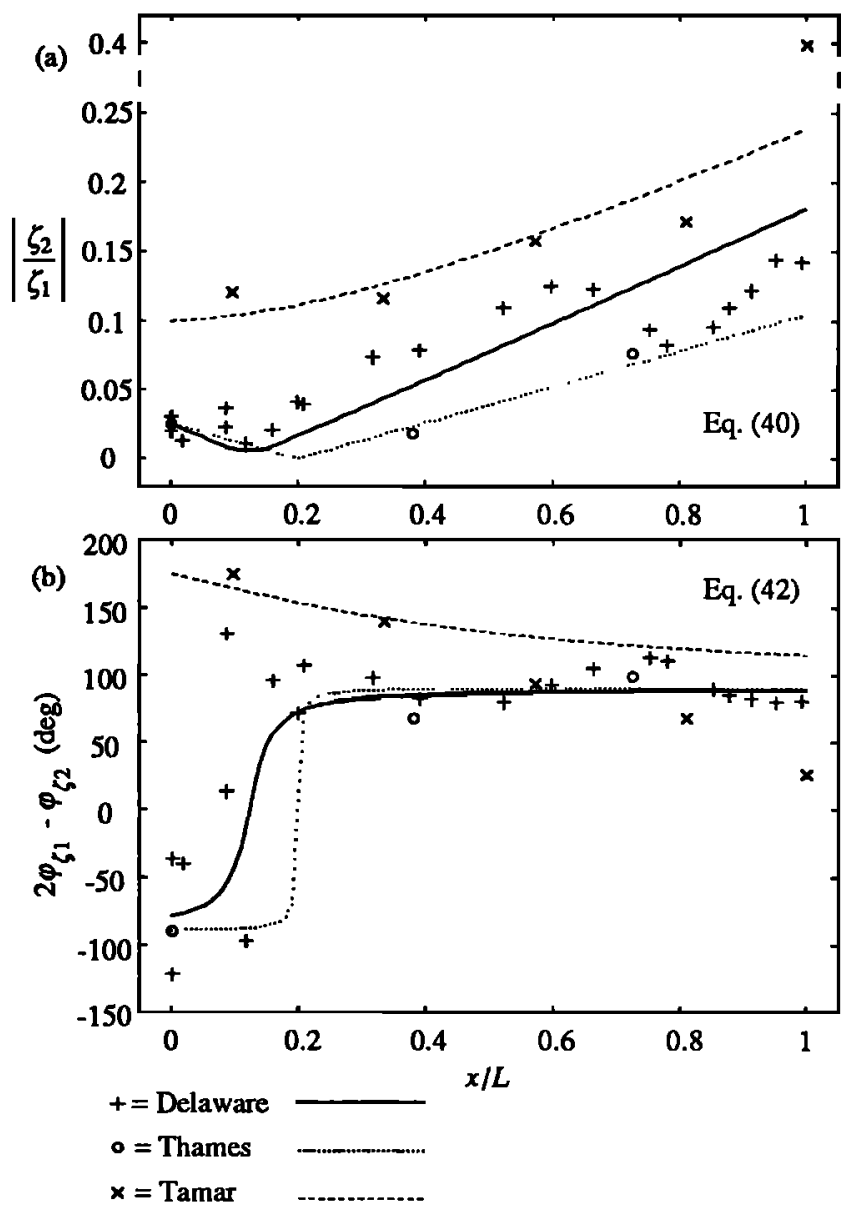

Figure 7. Observations of (a) amplitude and (b) phase of the second harmonic relative to the dominant component of tidal elevation as a function of distance along the Delaware [Parker, 1984], Tamar [George, 1975], and Thames [Hunt, 1964], along with predictions given by the second-order solution.

Otherwise $\gamma$ is derived from values of $\varepsilon_{h}$ and $\varepsilon_{b}$ displayed in Table 1. A good fit to the observations is achieved with $\delta=$ 0.6 for the Tamar, $\delta=0.3$ for the Thames, and $\delta=0$ for the Delaware (which gives $\gamma \approx 1.1,0.19$, and 0.11 for the three estuaries, respectively). Thus the net nonlinear effect of time variations in depth in the friction term appears to decrease as $\varepsilon_{h}$ decreases and as acceleration becomes more important in the momentum balance. In general, nonlinear friction appears to be less important than nonlinear continuity, a result which is consistent with the findings of Parker [1984, 1991].

Equations (40) and (42) capture both the order of magnitude and the along-channel trend in $\left|\zeta_{2} / \zeta_{1}\right|$ and $2 \ddot{\varphi}_{\zeta 1}$ $\varphi_{\zeta 2}$. In particular, the analytic solutions capture the transition from $\zeta_{2}$ dominated by external forcing to $\zeta_{2}$ dominated by internal nonlinear generation. Observed $2 \varphi_{\zeta 1}$ $\varphi_{\zeta 2}$ asymptotically approaches $90^{\circ}$ in the inner Thames and Delaware as predicted by (42) and (43). Equations (40) and (42) underpredict observed $\zeta_{\zeta_{2}} / \zeta_{1} \mid$ and overpredict observed $2 \varphi_{\zeta 1}-\varphi_{\zeta_{2}}$ in the innermost Tamar because the tidal curve is kinematically truncated by the elevation of the channel bottom [George, 1975]. Harmonic analyses of severely truncated tidal curves typically produce large $\zeta_{2}$ to $\zeta_{1}$ ratios 
$(\sim 0.3-0.4)$ and low $\zeta_{2}$ to $\zeta_{1}$ relative phases $\left(\sim 30^{\circ}\right)$ [Speer et al., 1991]. However the kinematics of this process are not represented by the dynamics of (40) to (43).

4.2.4. Velocity asymmetry and morphodynamic feedback. If the rising tide is of shorter duration than the falling tide $(\gamma>0)$ and high and low water nearly coincide with slack water, then continuity arguments require that velocity during the flood be greater than velocity during the ebb. Similarly, if the falling tide is of shorter duration $(\gamma<$ 0 ), then velocity during the ebb will be greater. Because tidally generated residual currents are negligible in strongly convergent channels, flood or ebb dominance is represented at second order entirely by the superposition of $u_{2}$ and $u_{1}$. Explicit analytic expressions for $\left|u_{2} / u_{1}\right|$ and $2 \varphi_{u 1}-\varphi_{u 2}$, which can be derived from (34) and (35), are much messier than analogous expressions for $\left|\zeta_{2} / \zeta_{1}\right|$ and $2 \varphi_{\zeta 1}-\varphi_{\zeta 2}$. Because of their complicated form and the dearth of highquality observations with which to compare them, they are not presented here.

Because of the difficulties inherent in simultaneously measuring velocity over an entire cross section, time series of cross-sectionally averaged velocity are available only for the Tamar [Uncles et al., 1985]. Although no simultaneous observations of elevation are available to provide appropriate values of $\zeta_{1}$ and $\zeta_{2}$ for forcing at $x=0$, Figure 8 nonetheless compares $u_{2}+u_{1}$ (calculated from (34) and (35)) to the available observations of cross-sectionally averaged velocity. The analytic solution uses the same geometric and forcing parameters listed in Table 1 and used in Figure 7. The velocity observations are from individual nonsynoptic spring tidal cycles and include the effects of runoff and diumal inequalities. Furthermore, it is not clear that reference tidal phase is consistent among the three time series. Despite these limitations, the analytic solution for $u$ captures the following important features of the observed time series: (1) the correct overall degree of distortion, (2) weak ebb dominance near the mouth of the estuary, and (3) increasingly strong flood dominance with increased landward distance. The analytic solution disagrees with the time series most strongly around low-water slack tide, which is when kinematic truncation of the tidal cycle by the channel bottom is most significant.

Flood dominance in the inner portions of shallow, strongly convergent tidal channels may provide a morphodynamic explanation for why the amplitude growth factor $\left(\mu=\varepsilon_{\omega}-\varepsilon_{A}\right)$ tends to be slightly greater than zero along these systems (see Table 1). Flood dominance tends to transport sediment in a landward direction, favoring channel shoaling as sediment collects in the inner estuary [e.g., Aubrey, 1986]. Thus flood-dominant tidal channels will not attain a stable form over the long term unless a physical mechanism simultaneously exists which favors seaward transport. As discussed in section 4.2.2, systemwide gradients in the magnitude of maximum velocity also cause net transport of sediment due to scour lag and settling lag [Postma, 1967]. If $\mu>0$, velocity amplitude will increase landward, and scour lag and settling lag will favor seaward sediment transport.

One might imagine the morphologic evolution of a shallow, exponentially convergent tidal channel to proceed
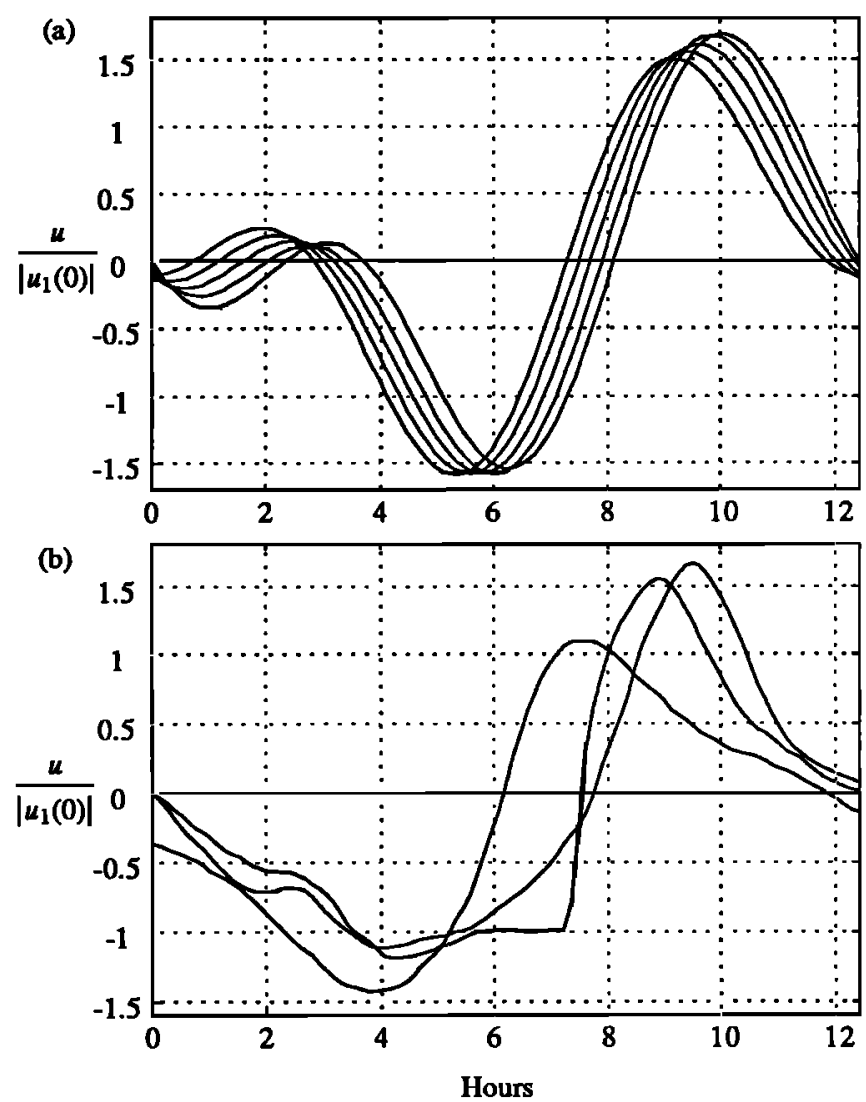

Figure 8. Time series of cross-sectionally averaged velocity at spring tide for the Tamar, normalized by the amplitude of $u_{1}$ at $x$ $=0$ (or the most seaward cross section). (a) Analytic solutions at $x=0,0.2,0.4,0.6$ and 0.8 ; (b) observations from Uncles $e t$ al. [1985] taken at $x=0.10,0.38$, and 0.57 .

as follows: Assuming the asymmetry factor is positive, flood dominance will cause sediment to collect in the inner portion of the estuary. If $\mu<0$, scour lag and settling lag will enhance the landward movement of sediment. As sediment collects in the landward reaches of the estuary, however, the cross section will become more strongly convergent and $\mu$ will increase. Eventually the amplitude growth factor will become positive and the amplitude of tidal velocity will increase in a landward direction. With $\mu>0$, scour lag and settling lag will tend to move sediment in a seaward direction. The larger the asymmetry factor, the larger $\mu$ will grow before a balance is reached between landward and seaward transport. The estuaries examined in this study support this relationship: $\gamma$ and $\mu$ are both largest for the Tamar, which is also the one channel where the amplitude of tidal velocity unambiguously increases in a landward direction. Morphodynamic feedback between spatial and temporal asymmetries in bottom stress is discussed in more detail by Friedrichs [1993] and is the subject of ongoing research.

\section{Summary and Conclusions}

A scaling of the continuity equation appropriate to shallow, strongly convergent channels (such as the Thames and Tamar in the United Kingdom and the Delaware in the United States) indicates gradients in tidal discharge are dominated at first order by gradients in cross-sectional area. 
Finite amplitude effects and gradients in velocity due to tidal phase enter at second order, and gradients in the amplitude of tidal velocity enter at third order. A scaling of the momentum equation then indicates that the first-order balance will be between pressure gradient and friction if the ratio of the observed phase speed to the frictionless wave speed is order one. Local acceleration contributes to momentum at second order, and advective acceleration enters only at fourth order.

Applying the above scaling, the first-order governing equation for elevation in strongly convergent channels becomes a first-order wave equation, in contrast to the classical second-order equation which results from low friction and a prismatic channel. The first-order wave equation allows wave propagation only in the incident direction and is inconsistent with the presence of a reflected wave. The first-order solutions for elevation and velocity are both constant amplitude waves with velocity leading elevation by $90^{\circ}$. Like a classical progressive wave, phase increases linearly along channel, and like a classical standing wave, currents are slack near high and low water. Yet unlike either classical wave, the dynamic balance is strongly frictional. Furthermore, the solutions are independent of the length of the estuary, in sharp contrast to the length-sensitive quarter-wave resonance of classical tidal estuary theory.

Second-order solutions for elevation and velocity at the dominant frequency are also unidirectional waves with the same phase speed as the first-order solution. However, the amplitudes of elevation and velocity are both modulated by $e^{\mu k x}$, where $k$ is the first-order wave number, and $\mu$ is the amplitude growth factor. The amplitude growth factor synthesizes the partially canceling effects of (1) local acceleration relative to friction and (2) limited convergence. If inertia is finite and convergence is strong, energy is concentrated along channel and amplitude increases with distance $(\mu>0)$. If inertia is weak and convergence is limited, friction causes amplitude to decay $(\mu<0)$. Because $\mu$ also determines the growth or decay of velocity (and bottom stress) with distance, $\mu$ should be near zero in channels which are morphologically stable. The secondorder solution indicates that when $\mu=0$, the wave speed (c) is exactly equal to the frictionless wave speed $\left(c_{0}\right)$. This explains why $c$ is usually close to $c_{0}$ in convergent channels, despite the dominance of friction at first order.

Compact second-order solutions for harmonics of elevation and velocity are both scaled by the tidal asymmetry factor, $\gamma$. The asymmetry factor synthesizes the partially canceling effects of (1) time variations in channel depth, which slow the propagation of low water and (2) time variations in estuary width, which slow the propagation of high water. If $\gamma>0$ (as is the case for the Thames, Tamar, and Delaware), the wave crest propagates faster than the trough, and the rising tide is of shorter duration. Away from the immediate vicinity of the channel mouth where external forcing may dominate, the elevation harmonic grows linearly with distance along channel, and the relative phase of the elevation harmonic asymptotically approaches $90^{\circ}$. If the tide is shorter rising, conservation of mass requires the tide within the inner estuary to be flood dominated. Since flood dominance favors the collection of sediment in the inner estuary, channel form may not be stable over the long term unless a physical mechanism simultaneously exists which favors seaward sediment transport. A slightly positive amplitude growth factor (which is the case over most of the Thames, Tamar and Delaware) may provide such a mechanism by increasing bottom stress in the inner estuary.

\section{Appendix: Small Terms Used in Scaling Governing Equations and in Approximating Solution}

Formally second order, $\mathrm{O}(\varepsilon)$ quantities are as follows:

$$
\begin{gathered}
\varepsilon_{h}=a / \bar{h} \\
\varepsilon_{b}=1-w / \bar{b} \\
\varepsilon_{U}=\left|L_{A} / L_{U^{1}}\right|^{1 / 2} \\
\varepsilon_{A}=k L_{A} \\
\varepsilon_{a}=\left|L_{A} / L_{a}\right| 1 / 2 \\
\varepsilon_{\omega}=\varepsilon_{A}\left(c / c_{0}\right)^{2}=\omega / r \\
\gamma=(1+\delta) \varepsilon_{h}-\varepsilon_{b} \\
\mu=\varepsilon_{\omega}-\varepsilon_{A}
\end{gathered}
$$

Other informally small quantities: $h / w ;\left(L_{A}-L_{b}\right) / L_{A} ; L_{A} / L$ ( $L_{A} / L$ is important only in vicinity of landward boundary).

\section{Notation}

$a$ amplitude of tidal elevation at $x=0$.

a2 amplitude of second elevation harmonic at $x=0$.

$A$ cross-sectional area of channel.

$\bar{A}$ overbar indicates time average (holds for all variables).

$b$ estuary width, including flats.

$c$ phase speed of tidal wave.

$c_{d}$ bottom friction drag coefficient.

$c_{0}$ frictionless shallow water wave speed.

$F$ friction term in momentum equation.

$g$ acceleration of gravity.

$h$ cross-sectionally averaged channel depth.

$h_{R}$ hydraulic radius.

${ }_{-j} j$ signifies value of variable $\_$for $j$ th channel segment.

$k$ tidal wave number.

$L$ length of tidal channel.

$L_{a} e$-folding length of variation in tidal amplitude.

$L_{A} e$-folding length of cross-sectional area convergence.

$L_{b}$ e-folding length of estuary width convergence.

$L_{U} e$-folding length of variation in velocity amplitude.

$L_{\theta}$ tidal wavelength.

$r$ linearized friction factor.

$t$ time.

$u$ cross-sectionally averaged velocity.

$u_{m} m$ th harmonic of tidal velocity.

$U$ amplitude of tidal velocity.

$w$ channel width.

$\boldsymbol{x}$ along-channel coordinate. 
$x_{b}$ location of tidal barrier.

$\gamma$ tidal asymmetry factor.

$\delta$ scales relative importance of nonlinear friction.

$\zeta$ tidal elevation.

$\zeta_{m} m$ th harmonic of tidal elevation.

$\theta_{2}$ phase of second elevation harmonic at $x=0$.

$\varepsilon$ signifies second-order term.

$\varepsilon_{a}{ }^{2}$ length scale ratio of convergence to amplitude change.

$\varepsilon_{A}$ length scale ratio of convergence to tidal wavelength.

$\varepsilon_{b}$ intertidal parameter.

$\varepsilon_{h}$ finite amplitude parameter.

$\varepsilon_{U}^{2}$ length scale ratio of convergence to velocity change.

$\varepsilon_{\omega}$ ratio of acceleration to pressure gradient or friction.

$\mu$ amplitude growth factor.

$\varphi_{u m}$ phase angle of $m$ th harmonic of tidal velocity.

$\varphi_{\zeta m}$ phase angle of $m$ th harmonic of tidal elevation.

$\omega$ radian tidal frequency.

Acknowledgments. Special thanks are extended to J. Boon, D. Jay, D. Lynch, O. Madsen, and J. Trowbridge who provided helpful comments on the manuscript. This work was supported by the National Science Foundation, under grant OCE 9102429. WHOI contribution 8422 .

\section{References}

Allen, G. P., J. C. Salomon, P. Bassoullet, Y. Du Penhoat, and C. De Grandpre, Effects of tides on mixing and suspended sediment transport in macrotidal estuaries, Sediment. Geol., $26,69-90,1980$.

Aubrey, D. G., Hydrodynamic controls on sediment transport in well-mixed bays and estuaries, in Physics of Shallow Estuaries and Bays, Coastal and Estuarine Studies vol.16, edited by J. van de Kreeke, pp. 245-258, Springer-Verlag, New York, 1986.

Chantler, A. G., The applicability of regime theory to tidal watercourses, J. Hydraul.Res., 12, 181-191, 1974.

Dronkers, J. J., Tidal Computations in Rivers and Coastal Waters, 518 pp., North-Holland, New York, 1964.

Friedrichs, C. T., Hydrodynamics and morphodynamics of shallow tidal channels and intertidal flats, Ph.D. thesis, 218 pp., WHOI/MIT Joint Program in Oceanogr., Mass. Inst. of Technol./Woods Hole Oceanogr. Inst., Woods Hole, Mass., 1993.

Friedrichs, C. T., and D. G. Aubrey, Non-linear tidal distortion in shallow well-mixed estuaries: A synthesis, Estuarine Coastal Shelf Sci., 27, 521-545, 1988.

Friedrichs, C. T., and O. S. Madsen, Nonlinear diffusion of the tidal signal in frictionally dominated embayments, $J$. Geophys. Res., 97, 5637-5650, 1992.

George, K. J., The tides and tidal streams of the Tamar estuary, Ph.D. thesis, 555 pp., Univ. of London, 1975.

Godin, G., Tides, 290 pp., Centro de Investigación Científica y de Educación Superior de Ensenada, Ensenada, Mexico, 1988.

Harleman, D. R. F., Tidal dynamics in estuaries, II: Real estuaries, in Estuary and Coastline Hydrodynamics, edited by A. T. Ippen, pp. 522-545, McGraw-Hill, New York, 1966.

Hunt, J. N., Tidal oscillations in estuaries, Geophys. J. $R$. Astron. Soc., 8, 440-455, 1964.

Ippen, A. T., Tidal dynamics in estuaries, I: Estuaries of rectangular section, in Estuary and Coastline Hydrodynamics, edited by A. T. Ippen, pp. 493-522, McGraw-Hill, New York, 1966.
Jay, D. A., Green's law revisited: Tidal long-wave propagation in channels with strong topography, J. Geophys. Res., 96, 20,585-20,598, 1991.

Kreiss, H., Some remarks about nonlinear oscillations in tidal channels, Tellus, 9, 53-68, 1957.

LeBlond, P. H., On tidal propagation in shallow rivers, $J$. Geophys. Res., 83, 4717-4721, 1978.

Le Floch, J., Propagation de la marée dans l'estuaire de la Seine et en Seine-Maritime, Sc.D. thesis, 507 pp., Univ. of Paris, 1961.

Lewis, R. E., and J. O. Lewis, Shear stress variations in an estuary, Estuarine Coastal Shelf Sci., 25, 621-635, 1987.

McDowell, D. M., and B. A. O'Connor, Hydraulic Behaviour of Estuaries, 292 pp., John Wiley, New York, 1977.

Münchow, A., A. K. Masse, and R. W. Garvine, Astronomical and nonlinear tidal currents in a coupled estuary shelf system, Cont. Shelf Res., 12, 471-498, 1992.

Nichols, M. M., and R. B. Biggs, Estuaries, in Coastal Sedimentary Environments, 2nd ed., edited by R. A. Davis, pp. 77-186, Springer-Verlag, New York, 1985.

Officer, C. B., Physical Oceanography of Estuaries (and Associated Coastal Waters), 465 pp., John Wiley, New York, 1976.

Parker, B. B., Frictional effects on the tidal dynamics of a shallow estuary, Ph.D. thesis, 292 pp., Johns Hopkins Univ., Baltimore, Md., 1984.

Parker, B. B., The relative importance of the various nonlinear mechanisms in a wide range of tidal interactions (review), in Tidal Hydrodynamics, edited by B. B. Parker, pp. 237-268, John Wiley, New York, 1991.

Perroud, P., The propagation of tidal waves into channels of gradually varying cross-section, Tech. Memo 112, 27 pp., U. S. Army Beach Erosion Board, Washington, D.C., 1959.

Postma, H., Sediment transport and sedimentation in the estuarine environment, in Estuaries, edited by G. H. Lauff, pp. 158-179, American Association for the Advancement of Science, Washington, D.C., 1967.

Prandle, D., A numerical model of the southern North Sea and River Thames, Rep. 4, 24 pp., Inst. of Oceanog. Sciences, Bidston Observ., Birkenhead, Cheshire, England, 1974.

Prandle, D., Modelling of tidal barrier schemes: An analysis of the open-boundary problem by reference to AC circuit theory, Estuarine Coastal Mar. Sci., 11, 53-71, 1980.

Prandle, D., and M. Rahman, Tidal response in estuaries, $J$. Phys. Oceanogr., 10, 1552-1573, 1980.

Robinson, I. S., L. Warren, and J. F. Longbottom, Sea-level fluctuations in the Fleet, an English tidal lagoon, Estuarine Coastal Shelf Sci., 16, 651-668, 1983.

Salomon, J. C., and G. P. Allen, Rôle sédimentologique de la marée dans les estuaries à fort marnage, Co. Fr. Pétrol. Notes Mém., 18, 35-44, 1983.

Shetye, S. R., and A. D. Gouveia, On the role of geometry of cross-section in generating flood-dominance in shallow estuaries, Estuarine Coastal Shelf Sci., 35, 113-126, 1992.

Speer, P. E., and D. G. Aubrey, A study of non-linear tidal propagation in shallow inlet/estuarine systems, II: Theory, Estuarine Coastal Shelf Sci., 21, 207-224, 1985.

Speer, P. E., D. G. Aubrey, and C. T. Friedrichs, Nonlinear hydrodynamics of shallow tidal inlet/bay systems, in Tidal Hydrodynamics, edited by B. B. Parker, pp. 321-339, John Wiley, New York, 1991.

Uncles, R. J., and J. A. Stephens, Distributions of suspended sediment at high water in a macrotidal estuary, J. Geophys. Res., 94, 14,395-14,405, 1989.

Uncles, R. J., R. C. A. Elliott, and S. A. Weston, Lateral distributions of water, salt and sediment transport in a partly mixed estuary, in Proceedings of the 19th International Conference on Coastal Engineering, edited by B. L. Edge, pp. 3067-3077, American Society of Civil Engineers, New 
York, 1985.

Uncles, R. J., R. C. A. Elliott, and S. A. Weston, Observed and computed lateral circulation paterns in a partly mixed estuary, Estuarine Coastal Shelf Sci., 22, 439-457, 1986.

Wallis, S. G., and D. W. Knight, Calibration studies concerning a one-dimensional numerical tidal model with particular reference to resistance coefficients, Estuarine Coastal Shelf Sci., 19, 541-562, 1984.

Weisman, R. N., G. P. Lennon, and F. E. Schuepfer, Resistance coefficient in a tidal channel, in Estuarine and Coastal Modeling, edited by M. L. Spaulding, pp. 123-131, American Society of Civil Engineers, New York, 1990.

Wright, L. D., J. M. Coleman and B. G. Thom, Processes of channel development in a high-tide-range environment: Cambridge Gulf-Ord River Delta, Western Australia, $J$. Geol., 81, 15-41, 1973.

D. G. Aubrey, Department of Geology and Geophysics, Woods Hole Oceanographic Institution, Woods Hole, MA 02543. (e-mail: aubrey@mud.whoi.edu)

C. T. Friedrichs, Virginia Institute of Marine Science, School of Marine Science, College of William and Mary, Gloucester Point, VA 23062-1346. (e-mail: cfried@ches.phys.vims.edu)

(Received July 1, 1993; revised October 8, 1993; accepted October 22, 1993.) 